This item was submitted to Loughborough's Research Repository by the author.

Items in Figshare are protected by copyright, with all rights reserved, unless otherwise indicated.

\title{
Benchmarking excited-state calculations using exciton properties
}

PLEASE CITE THE PUBLISHED VERSION

https://doi.org/10.1021/acs.jctc.7b01145

PUBLISHER

(c) American Chemical Society (ACS)

VERSION

AM (Accepted Manuscript)

PUBLISHER STATEMENT

This work is made available according to the conditions of the Creative Commons Attribution-NonCommercialNoDerivatives 4.0 International (CC BY-NC-ND 4.0) licence. Full details of this licence are available at: https://creativecommons.org/licenses/by-nc-nd/4.0/

\section{LICENCE}

CC BY-NC-ND 4.0

\section{REPOSITORY RECORD}

Mewes, Stefanie A., Felix Plasser, Anna Krylov, and Andreas Dreuw. 2019. "Benchmarking Excited-state Calculations Using Exciton Properties". figshare. https://hdl.handle.net/2134/32229. 


\title{
Benchmarking excited-state calculations using exciton properties
}

\author{
Stefanie A. Mewes, ${ }^{\dagger, \ddagger}$ Felix Plasser, ${ }^{\llbracket}$ Anna Krylov, ${ }^{\S}$ and Andreas Dreuw ${ }^{*, \dagger}$ \\ $\dagger$ Interdisciplinary Center for Scientific Computing, Ruprecht-Karls University, Im \\ Neuenheimer Feld 205A, D-69120 Heidelberg, GERMANY \\ $\ddagger$ Centre for Theoretical Chemistry and Physics, The New Zealand Institute for Advanced \\ Study (NZIAS), Massey University Albany, Private Bag 102904, Auckland 0745, NEW \\ ZEALAND \\ 9Institute for Theoretical Chemistry, Faculty of Chemistry, University of Vienna, \\ Währingstr. 17, A-1090 Wien, AUSTRIA \\ $\S$ University of Southern California Los Angeles, CA 90089-0482, USA \\ E-mail: dreuw@uni-heidelberg.de
}

January 11, 2018

\begin{abstract}
Benchmarking is an every-day task in computational chemistry, yet making meaningful comparisons between different methods is non-trivial. Benchmark studies often focus on the most obvious quantities such as energy differences. But to gain insight, it is desirable to explain the discrepancies between theoretical methods in terms of underlying wave functions and, consequently, physically relevant quantities. We present a new strategy of benchmarking excited-state calculations, which goes beyond excitation energies and oscillator strengths and involves the analysis of exciton properties based on the one-particle transition density matrix. By using this approach, we compare the
\end{abstract}


performance of many-body excited-state methods (equation-of-motion coupled-cluster and algebraic diagrammatic construction) and time-dependent density functional theory. The selected examples illustrate the utility of different exciton descriptors in assigning state character and explaining the discrepancies among different methods. The examples include Rydberg, valence, and charge-transfer states as well as delocalized excitonic states in large conjugated systems and states with substantial doubly excited character.

\section{Introduction}

One important task in quantum chemistry $^{1}$ is proper benchmarking of approximate computational methods against high-level, highly accurate ones. ${ }^{2-11}$ Such benchmark studies are motivated by the limited applicability of highly accurate methods to large systems due to unfavorable computational scaling of many-body theories leading to high computational demands. ${ }^{1}$ The necessity to employ computationally more efficient but less reliable methods ${ }^{12-14}$ together with limited experimental data undermine the credibility of computational protocols. In benchmarking excited-state calculations, the main (and often only) criterion is the excitation energy. While the excitation energy is certainly very important, the character of the underlying states should also be correctly described by approximate methods. Yet, the analysis of the underlying wave functions is often omitted, because comparing orbitals and amplitudes systematically and quantitatively is a non-trivial task. ${ }^{15}$ But exactly these details are crucial for understanding the differences in the physical description of excited states and can be a decisive factor in determining the domains of applicability of approximate models.

In this contribution, we benchmark excited-state calculations by exploiting the concept of excitons, i.e., correlated electron-hole pairs. ${ }^{16}$ The key quantity in the analysis is the one-particle transition density matrix, ${ }^{17}$ which affords a concise description of an electronic 
transition between the ground $\Phi^{0}$ and electronically excited $\Phi^{I}$ state:

$$
\begin{array}{r}
\gamma_{p q}^{0 I}=\left\langle\Phi^{0}\left|\hat{p}^{\dagger} \hat{q}\right| \Phi^{I}\right\rangle \equiv \gamma_{p q} \\
\gamma_{p q}^{I 0}=\left\langle\Phi^{I}\left|\hat{p}^{\dagger} \hat{q}\right| \Phi^{0}\right\rangle
\end{array}
$$

where $\hat{p}^{\dagger}$ and $\hat{q}$ are the creation and annihilation operators corresponding to $\phi_{p}$ and $\phi_{q}$ molecular orbitals. ${ }^{18}$ In the case of the configuration interaction singles (CIS) or similarly in Kohn-Sham time-dependent DFT (TDDFT), $\gamma_{p q}^{I 0}$ is equal to the wave function amplitudes, $\gamma_{p q}^{I 0}=\delta_{p a} \delta_{q i} C_{i}^{a}$, where, $C_{i}^{a}$ is a CIS coefficient corresponding to $\Phi_{i}^{a}$, a Slater determinant in which an electron is excited from occupied orbital $i$ to virtual orbital $a$. Thus, in the case of CIS/TDDFT wave functions/densities, the individual elements of $\gamma^{I 0}$ correspond to the weights of the electronic transitions between the respective molecular orbitals. Eq. (1) extends this simple molecular orbital picture of electronic transitions to general correlated wave functions: ${ }^{19,20} \gamma^{I 0}$ gives a map of one-electron transitions representing the changes between $\Phi^{I}$ and $\Phi^{0}$, which can be formulated as a linear combination of one-electron excitations:

$$
\left|\Phi^{I}\right\rangle=\sum_{p q} \gamma_{p q}^{I 0} \hat{p}^{\dagger} \hat{q}\left|\Phi^{0}\right\rangle+\text { higher excitations. }
$$

Using $\gamma_{p q}$, one can describe the transition density, $\rho^{t r}$ :

$$
\rho^{t r}(r)=\sum_{p q} \gamma_{p q} \phi_{p}(r) \phi_{q}(r)
$$

where $\phi_{p}$ and $\phi_{q}$ denote molecular orbitals. Plotting $\gamma_{p q}^{0 I}$ in spatial representation visualizes the exciton, i.e., the change in electronic distribution between the initial and final states. ${ }^{19-21}$ The transition density matrix, expressed in coordinate space, can be interpreted as exciton wave function: ${ }^{16,19,20}$

$$
\chi_{e x c}\left(r_{h}, r_{e}\right)=\sum_{p q} \gamma_{p q} \phi_{p}\left(r_{h}\right) \phi_{q}\left(r_{e}\right)
$$


where $r_{h}$ and $r_{e}$ denote hole and electron (particle) coordinates, as per eq. (3). Obviously, $\rho^{t r}(r)=\chi_{e x c}(r, r)$.

Using natural transition orbitals (NTOs),${ }^{22-25}$ the exciton can be represented in a very compact form:

$$
\chi_{e x c}\left(r_{h}, r_{e}\right)=\sum_{K} \sigma_{K} \psi_{K}^{h}\left(r_{h}\right) \psi_{K}^{e}\left(r_{e}\right)
$$

Here, orbitals $\psi_{K}^{h}\left(r_{h}\right)$ and $\psi_{K}^{e}\left(r_{e}\right)$ (representing hole and electron (particle) states) are obtained by singular value decomposition (SVD) of the transition density matrix:

$$
\begin{aligned}
\gamma^{0 I} & =\mathbf{V} \boldsymbol{\Sigma} \mathbf{U}^{T}, \\
\psi_{K}^{h}(r) & =\sum_{q} U_{q K} \phi_{q}(r), \\
\psi_{K}^{e}(r) & =\sum_{q} V_{q K} \phi_{q}(r),
\end{aligned}
$$

and index $K$ marks the NTO pair corresponding to the singular value $\sigma_{K}$. This representation of the electronic transitions removes the arbitrariness associated with a specific choice of molecular orbitals and describes the exciton in the most compact form. Orbital choice affects the wave function amplitudes (and, consequently, the resulting transition density matrices), such that a wave function with a single non-zero amplitude in one basis can be transformed into a wave function with multiple amplitudes with similar weights in another basis. SVD procedure produces the essential description of the transition, independent of orbital choice. Usually, only a small number of singular values are substantial, so eq. (6) represents the exciton wave function by using a (very) small number of terms. Using $\chi_{e x c}$, one can also compute a variety of exciton properties, which can serve as a basis for detailed analyses of excited states. ${ }^{16,19,20,26}$

The exciton-based analysis of the electronic transitions provides insight into the character of excited electronic states ${ }^{16,19,20,26}$ and offers several advantages for benchmarking:

1. It is independent from the method-dependent MO picture. 
2. It can be applied to any excited-state ansatz giving access to the one-particle transition density matrix.

3. It enables explicit benchmarking of electron-hole correlation phenomena, which are directly related to the physical description of excited states and crucial for the performance of quantum-chemical methods.

4. It facilitates state character assignment, as different types of excited states can be distinguished by the features that are easy to identify (for example, Rydberg states possess large electron sizes, charge-transfer states have large separations between the centroids of holes and electrons).

5. It affords detailed benchmarking of different methods, even when experimental data is absent.

While reduced density matrices and NTOs have been used in electronic structure for quite some time,${ }^{22-25}$ using them to compute properties of excitons is relatively new. ${ }^{16,19,20,26}$ Complementary to our approach, there exists a variety of descriptors based on orbital overlaps, ${ }^{27,28}$ changes in the density matrix or ${ }^{29,30}$ in the electron density. ${ }^{31}$ While these descriptors have been successfully employed for assessing the accuracy of TDDFT calculations, ${ }^{32,33}$ they have not yet been generalized to higher-level ab initio methods. Recently, one of us has introduced an alternative approach for comparing many-electron wave functions by calculating overlaps, ${ }^{34}$ quantifying variations in wave functions with respect to a reference wave function.

In the following, we illustrate that focusing on exciton properties opens a new route for a simple yet detailed benchmarking of excited states. In the methodology section, we briefly review the key equations and main features of the employed density analysis tools. We then proceed to a carefully chosen set of molecules that feature various types of excited states (results section). These systems, shown in Fig. 1, are formaldehyde, the push-pull system 4-( $N, N$-dimethylamino)benzonitrile (DMABN), all-trans octatetraene as a represen- 
tative for multiply excited states, hexa(thiophene) (6T) as a large conjugated system with delocalized $\pi \pi^{*}$ excited states, and magnesium porphyrin $(\mathrm{MgP})$ as large, biologically relevant compound. Two families of ab initio methods are compared: the equation-of-motion coupled-cluster singles doubles (EOM-CCSD) ${ }^{35-39}$ and the algebraic-diagrammatic construction for the polarization propagator $(\operatorname{ADC}(n))^{40-42}$ methods. In selected cases, we also employ time-dependent density functional theory ${ }^{12,43,44}$ in combination with a few, commonly used exchange-correlation functionals.

(a)

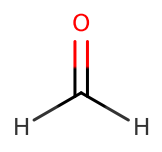

(d)

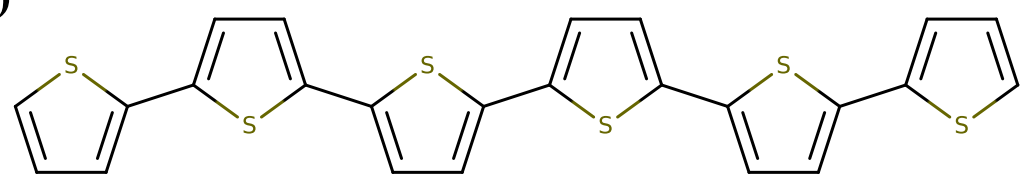

(c)<smiles>C=CC=CC=CC=C</smiles>

(e)

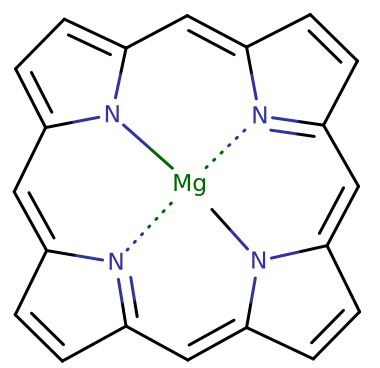

Figure 1: Molecules investigated in this work: (a) formaldehyde, (b) 4- $(N, N$ dimethylamino)benzonitrile (DMABN), (c) all-trans octatetraene, (d) hexa(thiophene) (6T), and (e) magnesium porphyrin $(\mathrm{MgP})$.

\section{Methodology}

In this section, we briefly review the key features of the analysis suite, libwfa, for the manuscript to be self-contained. Detailed further information can be found in Refs. 16,26,4549 and references therein; these papers explain various methodological aspects and also highlight special applications. 


\subsection{Transition density and exciton wave function}

The central quantity in our analysis is the exciton wave function $\chi_{e x c}$, which is identified with the one-particle transition density matrix between the ground $\left(\Phi^{0}\right)$ and an excited $\left(\Phi^{I}\right)$ state expressed in coordinate space. It is explicitly given as

$$
\chi_{e x c}\left(r_{h}, r_{e}\right)=\int \ldots \int \Phi^{0}\left(r_{h}, r_{2}, \ldots, r_{N}\right) \Phi^{I}\left(r_{e}, r_{2}, \ldots, r_{N}\right) d r_{2} \ldots d r_{N}
$$

where $N$ is the number of electrons and $r_{i}$ are the spatial-spin coordinates. Using the second-quantization definition of the transition density matrix (eq. (1)) allows one to ex-

press $\chi_{\text {exc }}\left(r_{h}, r_{e}\right)$ in terms of molecular orbitals (eq. (5)). Importantly, $\chi_{\text {exc }}$ contains all essential information needed to compute any one-electron interstate property. For example, the transition dipole moment is simply

$$
\left\langle\Phi^{0}|\hat{\mu}| \Phi^{I}\right\rangle=\int \chi_{e x c}(r, r) \mathbf{r} d r=\sum_{p q} \gamma_{p q} \mu_{p q}=\operatorname{tr}[\gamma \mu]
$$

where $\mu_{p q}=\left\langle\phi_{p}|\hat{\mu}| \phi_{q}\right\rangle$. Or, using NTOs:

$$
\left\langle\Phi^{0}|\hat{\mu}| \Phi^{I}\right\rangle=\sum_{K} \sigma_{K}\left\langle\psi_{K}^{h}|\hat{\mu}| \psi_{K}^{e}\right\rangle
$$

This representation allows one to express matrix elements between many-electron wave functions in terms of the matrix elements between orbitals, illuminating physical significance of excitons and NTOs through their relationship to the observables.

The exciton wave function can be further analyzed by calculating its expectation value with respect to an operator of interest:

$$
\langle\hat{O}\rangle_{e x c}=\frac{\left\langle\chi_{e x c}|\hat{O}| \chi_{e x c}\right\rangle}{\left\langle\chi_{e x c} \mid \chi_{e x c}\right\rangle}
$$


The denominator in eq. (12) is the squared norm of the exciton wave function

$$
\Omega \equiv\left\langle\chi_{\text {exc }} \mid \chi_{e x c}\right\rangle=\sum_{p q} \gamma_{p q}^{2}=\|\gamma\|^{2}
$$

As per eq. (13), $\Omega$ quantifies the amount of single excitation character of the $\Phi^{0} \rightarrow \Phi^{I}$ transition and provides an upper bound to the interstate matrix elements. ${ }^{19,50,51}$ Being orbitalinvariant, this quantity is ideal for comparing the single excitation character among different methods, in contrast to wave function amplitudes.

\subsection{Exciton descriptors}

Exciton wave function can be characterized by computing its spatial and statistical properties according to eq. (12). To do so, one first computes the multipole moments of the exciton wave function and then combines them to obtain quantities of interest. Multipole moments can be evaluated according to:

$$
\left\langle x_{h}^{k} x_{e}^{l}\right\rangle_{\text {exc }}=\frac{1}{\Omega} \operatorname{tr}\left(\gamma^{I 0} \mathbf{M}_{x}^{(k)} \gamma^{0 I} \mathbf{M}_{x}^{(l)}\right)
$$

where $\mathbf{M}_{x}^{(k)}$ denotes the $k$-order multipole matrix for coordinate $x$, whose components are given as:

$$
M_{x, p q}^{(k)}=\int \phi_{p}(r) x^{k} \phi_{q}(r) d r
$$

The above equations can also be formulated in the basis of atomic orbitals. By combining different multipole moments to compute physically meaningful exciton descriptors, we exploit their interpretive power in the context of excited-state analysis. In the following, we discuss selected descriptors available in Q-Chem ${ }^{52,53}$ and highlight their capabilities.

To illustrate exciton descriptors suitable to quantify the amount of charge-transfer character, consider the vectorial distance between the centroids of electron and hole distributions 
(a)

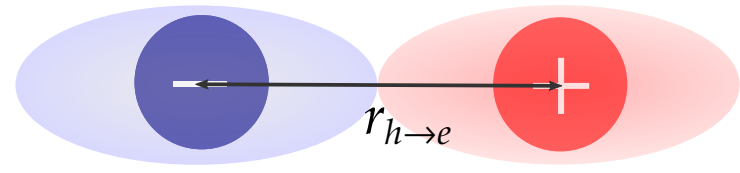

(b)

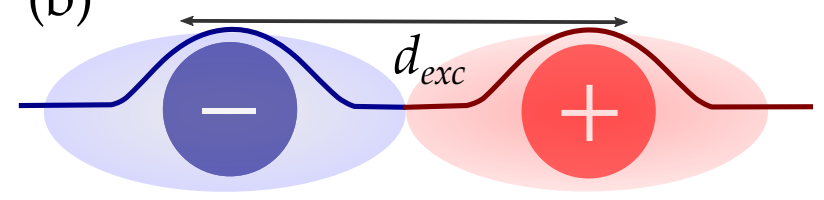

(c)

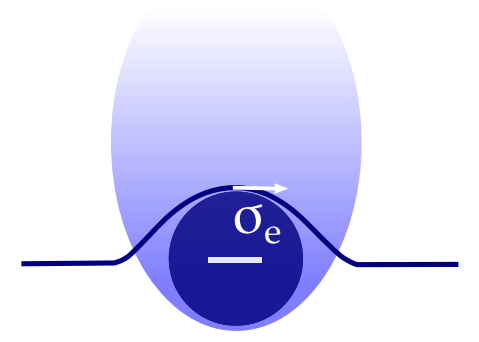

(d)

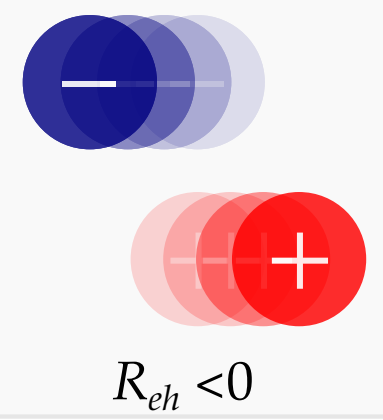

(e)

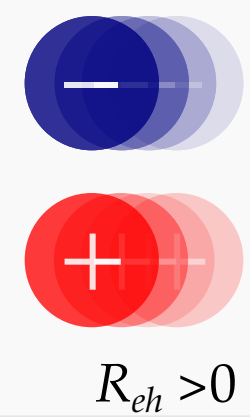

Figure 2: (a) Vectorial electron-hole distance $d_{h \rightarrow e},(\mathrm{~b})$ exciton size $d_{e x c}$ (rms electron-hole distance), (c) electron size $\sigma_{e}$ (rms deviation from the centroid of the electron density), (d) negative electron-hole correlation $R_{e h}<0$, i.e., dynamical charge avoidance, and (e) positive electron-hole correlation $R_{e h}>0$, i.e., joint electron-hole motion as bound exciton.

given as

$$
d_{h \rightarrow e}=\frac{1}{\Omega}\left|\left\langle\chi_{e x c}\left|r_{h}-r_{e}\right| \chi_{e x c}\right\rangle\right| \equiv\left|\left\langle r_{h}-r_{e}\right\rangle_{e x c}\right|,
$$

shown in Fig. 2(a). This quantity can be interpreted as an average distance between hole and electron (particle). A complementary measure is the exciton size that quantifies the root-mean-square electron-hole separation shown in Fig. 2(b)

$$
d_{e x c}=\sqrt{\left\langle\left|r_{h}-r_{e}\right|^{2}\right\rangle_{e x c}} .
$$

This formulation takes into account the sizes of electron $\left(\sigma_{e}\right)$ and hole $\left(\sigma_{h}\right)$ distribution as well as their covariance (COV) (explicit expressions for these quantities are given below), and allows one to express the exciton size as

$$
d_{e x c}=\sqrt{d_{h \rightarrow e}^{2}+\sigma_{h}^{2}+\sigma_{e}^{2}-2 \times \operatorname{COV}\left(r_{h}, r_{e}\right)} .
$$


While $d_{h \rightarrow e}$ quantifiespermanent charge-transfer character, the exciton size allows one to also characterize charge-resonance (or dynamic charge transfer). ${ }^{16,54}$ In analogy to the exciton size, the electron and hole sizes are defined as

$$
\sigma_{h}=\sqrt{\left\langle r_{h}^{2}\right\rangle_{e x c}-\left\langle r_{h}\right\rangle_{e x c}^{2}},
$$

quantifying the root-mean-square deviation of the hole or electron distribution with respect to their centroids shown in Fig. 2(c). These quantities are particularly useful for distinguishing between different types of excited states, e.g., Rydberg states, core-excited states ${ }^{55}$ etc. The compactness of electron and hole distributions is also related to correlation phenomena. ${ }^{56}$

Let us now discuss the statistical properties of the exciton wave function. The two following descriptors quantify linear correlation between electron and hole, i.e., the covariance and the correlation coefficient

$$
\operatorname{COV}\left(r_{h}, r_{e}\right)=\left\langle r_{h} \cdot r_{e}\right\rangle_{e x c}-\left\langle r_{h}\right\rangle_{e x c} \cdot\left\langle r_{e}\right\rangle_{e x c}
$$

The electron-hole correlation coefficient is simply the covariance normalized by the product of electron and hole sizes

$$
R_{e h}=\frac{\operatorname{COV}\left(r_{h}, r_{e}\right)}{\sigma_{h} \sigma_{e}} .
$$

A positive value of $R_{e h}$ identifies a bound exciton, because a change in hole position induces a change in electron position in the same direction, i.e., they move together in a correlated way. Conversely, a negative value indicates that electron and hole avoid each other in space, i.e., that they are anti-correlated. A correlation coefficient of zero corresponds to independent electron and hole quasi-particles (a situation typical for small molecules). We note that all exciton descriptors can be expressed in terms of NTOs, ${ }^{22-24}$ similarly to eq. (11). A detailed discussion of this relation can be found in Ref. 26 . 


\section{Computational details}

All calculations were performed with the Q-Chem ${ }^{52,53}$ electronic structure package. NTOs were visualized using Jmol.

Formaldehyde. The geometry was optimized at the RI-MP(2)/cc-pVTZ level of theory yielding $C_{2 v}$ symmetry. Excited-state calculations were carried out with the ADC ${ }^{40-42}$ method for the polarization propagator at the second and third order of perturbation theory as well as with the EOM-CCSD method for excitation energies (EOM-EE-CCSD). ${ }^{35-37,57}$ $\operatorname{ADC}(2)$ and $\operatorname{ADC}(3)$ were used in their standard variants in combination with the Ahlrichs' $\mathrm{SV}(\mathrm{P})$ and SVP basis sets, and in the Resolution-of-Identity approximation ${ }^{58}$ (RI) in combination with Ahlrichs' SV, ${ }^{59}$ SV(P) and SVP, and Dunning's cc-pVDZ, cc-pVTZ, aug-ccpVDZ, and aug-cc-pVTZ basis sets. ${ }^{60,61}$ EOM-CCSD was used in its canonical variant (no RI) for all basis sets.

DMABN. Six structures were considered: the fully relaxed, ground-state geometry (GS) optimized at the RIMP(2)/cc-pVDZ level of theory and five excited-state geometries, LE, CT0, CT45, CT90, CTP, optimized with RI-ADC(2)/cc-pVDZ. The LE and CTP structures were fully relaxed. For the CT geometries, constrained optimization was carried out with the twisting angle between the benzene ring and the dimethylamino group constrained to $0^{\circ}$ (CT0), 45 $(\mathrm{CT} 45)$, and $90^{\circ}$ (CT90). All optimizations were carried out in vacuum. Further details can be found in Ref. 62. Excited-state calculations were performed using the canonical variants of $\mathrm{ADC}(2) / \mathrm{cc}-\mathrm{pVDZ}, \mathrm{ADC}(3) / \mathrm{cc}-\mathrm{pVDZ}$ and EOM-CCSD/cc-pVDZ in vacuum. Calculating solvent effects, a state-specific polarizable continuum model (IEF-PCM) ${ }^{63}$ was employed in combination with standard $\mathrm{ADC}(2) / S S$-PCM/cc-pVDZ available in Q-Chem 5.0. The solvents cyclohexane (cHex, $\left.\varepsilon=1.89, n^{2}=1.88\right)$ and acetonitrile (MeCN, $\varepsilon=36.7$, $n^{2}=1.81$ ) were employed (information about specific input parameters can be found in Ref. $62)$. 
Hexa(thiophene). The ground-state geometry was first optimized using the $\omega \mathrm{B} 97 / \mathrm{SV}(\mathrm{P})$ level of theory and then symmetrized using IQmol (the symmetrization resulted in very small changes in total energy). Excited-state geometry of the $\mathrm{S}_{1}$ state was optimized using CAMB3LYP/cc-pVDZ. Excited-state calculations for vertical excitations were carried out at the RI-ADC(2), RI-EOM-CCSD (with core electrons frozen), and CIS ab initio levels of theory, and at the full-time-dependent density functional theory ${ }^{12,43,44}$ (TDDFT) and Tamm-Dancoff approximation $^{64}$ (TDA) levels employing BLYP, B3LYP, CAM-B3LYP exchange-correlation functionals. The three functionals feature different amounts of non-local orbital exchange: the BLYP functional (0\% of exact exchange) is a representative of local generalized-gradientapproximation-type (local GGA) functionals, the B3LYP functional is a global hybrid functional (21\% of exact exchange), ${ }^{65,66}$ and CAM-B3LYP is a long-range corrected method (19-65\% of exact exchange). ${ }^{67}$ All calculations were performed using Ahlrichs' SV(P) basis set. In addition, RI-EOM-CCSD calculations with aug-cc-pVDZ were carried out.

All-trans octatetraene. The ground-state geometry was optimized using CCSD(T)/ccpVTZ level of theory yielding $C_{2 h}$ symmetry. We note that high level of theory is essential to correctly describe the electronic structure of the ground state and to obtain accurate carboncarbon distances for the conjugated system. ${ }^{68}$ Excited-state calculations were carried out with RI-ADC(2), RI-ADC(3), RI-EOM-CCSD, as well as with TD-DFT/TDA using BLYP, B3LYP, and CAM-B3LYP $x c$-functionals employing Dunning's cc-pVTZ basis.

Magnesium(II)porphyrin. The ground-state geometry was optimized with RIMP(2)/TZVP, followed by IQmol symmetrization. Excited-state calculations were performed with RIADC(2)/SV(P), RI-EOM-CCSD/SV(P), RI-EOM-CCSD/cc-pVDZ, RI-EOM-CCSD/cc-pVTZ, $\operatorname{ADC}(3) / \mathrm{SV}(\mathrm{P})$, and CIS/SV $(\mathrm{P})$ as well as with TDDFT/TDA employing BLYP, B3LYP, and CAM-B3LYP as $x c$-functionals with Ahlrichs' SV(P) and TZVP basis sets.

Cartesian coordinates for all structures are given in SI. We note that Q-Chem does not follow the standard Mulliken convention ${ }^{69}$ for molecular orientation, such that the labels of some irreps are flipped. ${ }^{70}$ All raw numbers shown in figures are given in SI. 


\section{Results and discussion}

We now proceed to examine different types of excited states, focusing on exciton descriptors that deliver information relevant to each case. We illustrate which descriptors can aid the benchmark procedure and how they can inform us about methodological aspects.

\subsection{Valence and Rydberg states in formaldehyde}

A popular benchmark molecule, ${ }^{71,72}$ formaldehyde demonstrates the utility of exciton descriptors in distinguishing Rydberg and locally excited states. ${ }^{15}$ Rydberg states have relatively small hole sizes $\sigma_{h}$ and large electron sizes $\sigma_{e}$. In contrast, electron and hole sizes of locally excited states are rather similar. Table 1 shows excitation energies, term labels, and Table 1: Excited states of formaldehyde: irreducible representations, characters and excitation energies $(\mathrm{eV})$.

\begin{tabular}{|lll|lll|lll|}
\hline & & & \multicolumn{3}{|c|}{ cc-pVTZ } & \multicolumn{3}{c|}{ aug-cc-pVTZ } \\
state & character $^{a}$ & exp $^{a}$ & $\mathrm{ADC}(3)$ & $\mathrm{ADC}(2)$ & EOM-CCSD & ADC(3) & ADC(2) & EOM-CCSD \\
\hline $1^{1} \mathrm{~A}_{2}$ & $n \rightarrow \pi^{*}$ & 4.1 & 3.92 & 4.01 & 4.07 & 3.90 & 3.92 & 4.02 \\
$1^{1} \mathrm{~B}_{1}$ & $n \rightarrow 3 s$ & 7.13 & 8.77 & 7.66 & 8.35 & 7.63 & 6.52 & 7.24 \\
$2^{1} \mathrm{~B}_{1}$ & $n \rightarrow 3 p$ & 7.98 & 10.53 & 9.94 & 9.36 & 8.46 & 7.54 & 8.12 \\
$2^{1} \mathrm{~A}_{1}$ & $n \rightarrow 3 p$ & 8.14 & $(9.23)^{b}$ & 9.37 & 9.82 & 8.62 & 7.49 & 8.22 \\
$1^{1} \mathrm{~B}_{2}$ & $\sigma \rightarrow \pi^{*}$ & 9.0 & 9.20 & 9.29 & 10.28 & 9.18 & 9.18 & 9.30 \\
$3^{1} \mathrm{~A}_{1}$ & $\pi \rightarrow \pi^{*}$ & 10.7 & $(9.81)^{b}$ & 10.22 & $(10.57)^{c}$ & 9.06 & $(9.48)^{d}$ & 9.68 \\
\hline
\end{tabular}

${ }^{a}$ Experimental data and state assignment is from Ref. 71 .

${ }^{b}$ Substantial state mixing and double excitation character.

${ }^{c}$ State has $53 \%$ Rydberg character and $30 \% \pi \rightarrow \pi^{*}$.

${ }^{d}$ State has $64 \%$ Rydberg character and $25 \% \pi \rightarrow \pi^{*}$.

state characters for six singlet excited states of formaldehyde. ${ }^{71}$ The experimental values are compared with the computational results obtained with $\mathrm{ADC}(3), \mathrm{ADC}(2)$ and EOM-CCSD in combination with the cc-pVTZ and aug-cc-pVTZ basis sets. As expected, the cc-pVTZ basis is not sufficient for describing Rydberg states, leading to large errors in excitation energies. The results improve considerably in the aug-cc-pVTZ basis set: the state ordering is correct in almost all cases and the errors against the experimental values are much smaller. Using specialized basis sets ${ }^{74}$ for the description of Rydberg states may further improve the results, however, for the purpose of this study the obtained results suffice. Using this initial 
assessment of the accuracy of the methods, we now proceed to detailed analysis based on exciton descriptors.
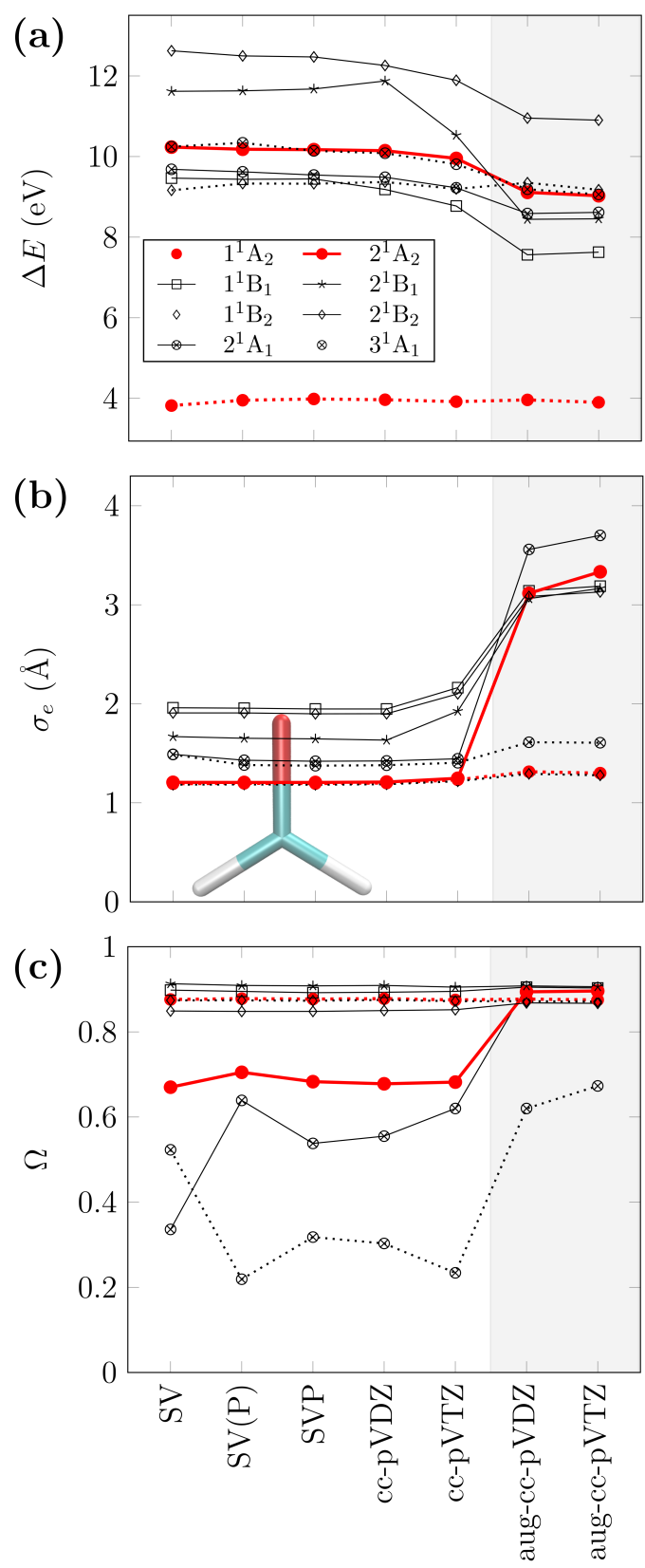

Figure 3: Excited states in formaldehyde. (a) Excitation energies $(\Delta E$, eV), (b) electron size $\left(\sigma_{e}, \AA\right)$, and (c) single excitation character $(\Omega)$ of the first two excited states of each irrep calculated by $\operatorname{ADC}(3)$ with different basis sets. The legend in (a) applies to all diagrams. The inset of the formaldehyde structure in (b) shows the size of the molecule.

Fig. 3 shows the ADC(3) results for the first two singlet excited states of each irrep. Two 
states are highlighted in red, $1^{1} \mathrm{~A}_{2}$ and $2^{1} \mathrm{~A}_{2}$, which we discuss in more detail below. Fig. 3(a) shows the changes in excitation energies with respect to the basis sets. The following hierarchy of basis sets is considered (from left to right): first, the polarization of the second-row atoms is included; second, basis is extended from double- $\zeta$ to triple- $\zeta$; third, diffuse functions are included (for both double- $\zeta$ and triple- $\zeta$ bases). While the excitation energies remain constant for almost all states for the first five basis sets (with a few exceptions), significant changes occur upon the augmentation. This is expected because diffuse functions are mandatory for the correct description of Rydberg states. ${ }^{15}$ Although more compact valence states do not require diffuse functions, they can mix and interact with Rydberg states, especially when density of states is high, ${ }^{15}$ as it happens around $9 \mathrm{eV}$ in formaldehyde. Consequently, locally excited states in this energy range also become stabilized in the augmented basis sets (black, dotted lines). This example illustrates that the dependence of excitation energy on the basis set is not sufficient for distinguishing Rydberg and valence states. Here, the utility of exciton analysis becomes obvious.

Fig. 3(b) and Fig. S1(b) in SI illustrate the effect of the basis set on the excited-state wave functions by considering electron and hole sizes (eq. (19)). While the hole sizes (Fig. S1(b) in SI) vary between 1.0 and $1.3 \AA$ (and only two excited states show a noticeable increase of $\sim 0.2 \AA$ upon the inclusion of diffuse functions), the electron sizes change dramatically for the Rydberg states (Fig. 3(b)). This behavior can be contrasted with almost constant values of $\sigma_{e}$ of the locally excited states $\left(1^{1} \mathrm{~A}_{2}, 1^{1} \mathrm{~B}_{2}, 3^{1} \mathrm{~A}_{1}\right)$. Thus, $\sigma_{e}$ is a key descriptor able to differentiate between valence and Rydberg states.

Fig 3(c) presents another important property, $\Omega$, which quantifies the amount of single excitation character in the excited-state wave functions. For a primarily singly excited state, $\Omega \approx 1$. We observe that in small (not augmented) basis sets, some states (two ${ }^{1} \mathrm{~A}_{1}$ states and $\left.2^{1} \mathrm{~A}_{2}\right)$ show significantly smaller values of $\Omega$ at the $\operatorname{ADC}(3)$ level of theory, which indicates substantial doubly excited character. This doubly excited character disappears when diffuse functions are included, suggesting that this is an artifact of using small basis sets (this is 
similar to a well known phenomenon of valence-space CASSCF overestimating doubly excited character).

Let us now compare the exciton characters obtained using $\mathrm{ADC}(2)$ and EOM-CCSD wave functions with the aug-cc-pVTZ basis set (the results for the aug-cc-pVDZ basis are given in SI).

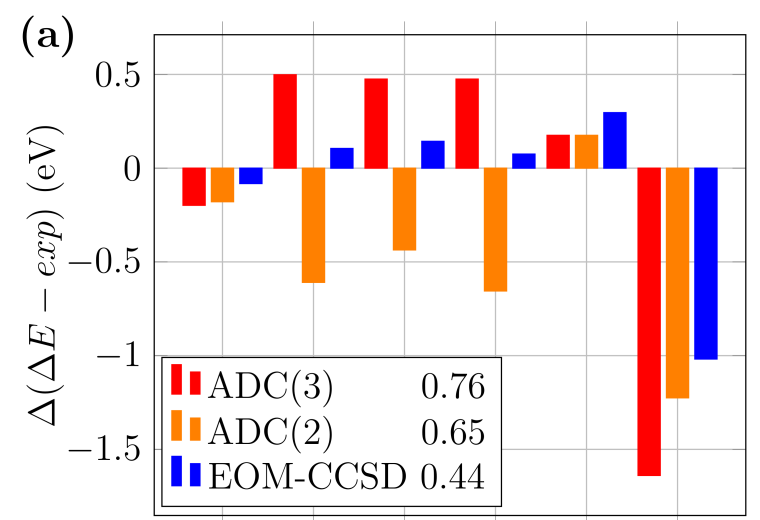

$1^{1} \mathrm{~A}_{2} 1^{1} \mathrm{~B}_{1} 2^{1} \mathrm{~B}_{1} 2^{1} \mathrm{~A}_{1} 1^{1} \mathrm{~B}_{2} 3^{1} \mathrm{~A}_{1}$ (b)

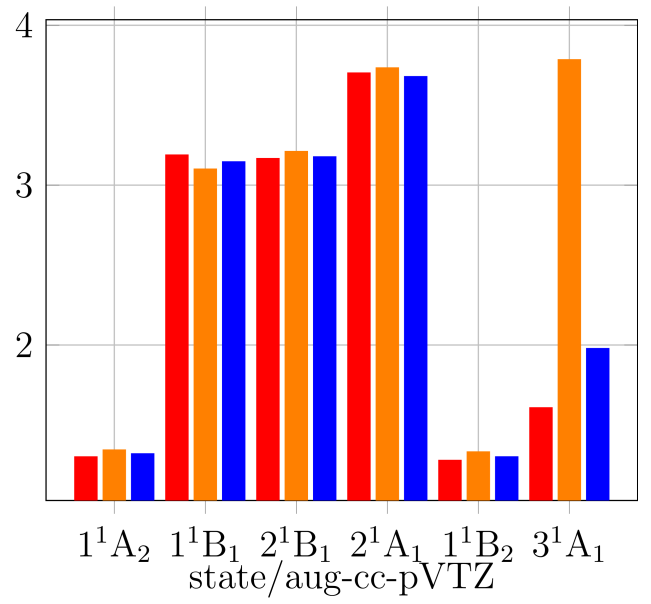

Figure 4: Excited states of formaldehyde. (a) Differences between experimental and computed excitation energies $\left(\Delta(\Delta E-e x p)\right.$, eV), and (b) electron sizes $\left(\sigma_{e}, \AA\right)$ of six excited states computed at the $\mathrm{ADC}(3), \mathrm{ADC}(2)$ and EOM-CCSD levels of theory employing the aug-cc-pVTZ basis set. Root-mean-square deviations $(\mathrm{eV})$ are displayed in legend.

Fig. 4 (a) presents the differences between experimental and computed excitation energies for the six excited states discussed above including the $\mathrm{ADC}(2), \mathrm{ADC}(3)$, and $\mathrm{EOM}-\mathrm{CCSD}$ levels of theory. The corresponding electron sizes are plotted in Fig. 4 (b). The deviations from the experimental values can be systematically explained in terms of the state characters. The ADC(2) method underestimates Rydberg excitation energies by about $0.5 \mathrm{eV}$, whereas $\mathrm{ADC}(3)$ overestimates energies of these states by $0.5 \mathrm{eV}$. EOM-CCSD yields the smallest errors for both valence and Rydberg states. One case that stands out is the $3^{1} \mathrm{~A}_{1}$ state for which all computational methods yield large errors in excitation energies. The electron sizes indicate that the state has some Rydberg character at the $\operatorname{ADC}(2)$ level, while it appears to be rather local in other methods. The analysis of single excitation character (Fig 3(c)) reveals that this state has some double excitation character. However, it seems that both 
$\mathrm{ADC}(3)$ and EOM-CCSD are underestimating the energy of this state.

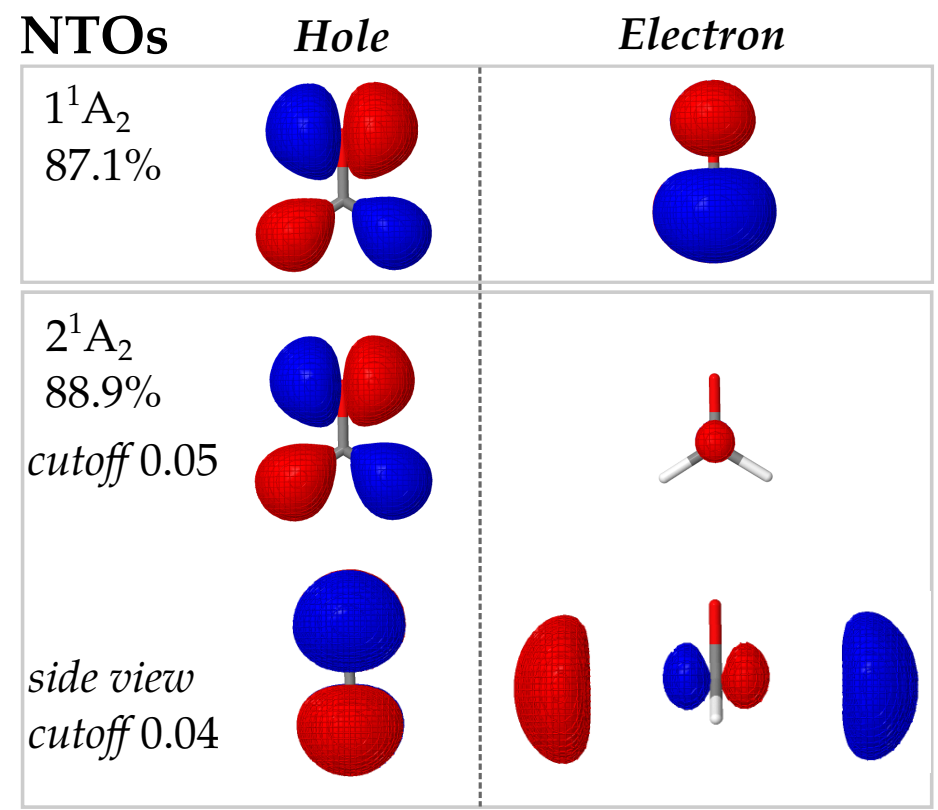

Figure 5: Natural transition orbitals of the $1^{1} \mathrm{~A}_{2}\left(n \rightarrow \pi^{*}\right)$ and $2^{1} \mathrm{~A}_{2}(n \rightarrow R y(3 p))$ excited states of formaldehyde calculated at the ADC(3)/aug-cc-pVTZ level of theory. Singular values are given in percentage. For the Rydberg states, two different isovalues are used (0.05 and 0.04 ).

Let us briefly highlight another issue relevant to Rydberg states. Fig. 5 shows NTOs for the $1^{1} \mathrm{~A}_{2}\left(n \rightarrow \pi^{*}\right)$ and $2^{1} \mathrm{~A}_{2}(n \rightarrow R y(3 p))$ excited states of formaldehyde. Both states are well described by a single NTO pair (the respective leading $\sigma^{2}$ values are 0.87 and 0.89 ). The visualization of the hole orbital is straightforward, however, accurate rendering of the electron orbital requires some care. While valence-like NTOs (such as $\pi^{*}$ electron orbital of the $1^{1} \mathrm{~A}_{2}$ state) can be adequately visualized using the same isovalues as used for the hole orbitals, the diffuse Rydberg orbitals need to be rendered using smaller isovalues. When using the same isovalue as for valence orbitals, the isosurface encloses only a small part of the electron density of much more diffuse Rydberg orbitals (this can be easily understood by comparing two normalized gaussians with different exponents, see Fig. S3 in Supplementary Information), which leads to a misleading picture, as illustrated by the middle row of Fig. 5 . When isovalue is adjusted to a lower value (as in the bottom row of Fig. 5), the true shape of the Rydberg orbital becomes visible. Using simple exciton descriptors characterizing electron 
sizes informs users about Rydberg character of the state, alerting them that a smaller isovalue needs to be used for rendering NTOs.

\subsection{Charge-transfer states in DMABN}

Charge-transfer states present a challenge for many excited-state methods; consequently, they are often used in benchmark studies, highlighting methodological issues. ${ }^{27,73}$ We consider 4-( N,N-Dimethylamino)benzonitrile (DMABN), a substituted benzene ring with donor and acceptor groups in para position, as a representative organic push-pull molecule. The photochemistry of DMABN is rather complex. This molecule features dual fluorescence in polar environments, ${ }^{75}$ where there are two fluorescence peaks, one at $350 \mathrm{~nm}(3.54 \mathrm{eV})$ and another at $475 \mathrm{~nm}(2.61 \mathrm{eV})$; the latter peak vanishes in gas phase. The origin of dual fluorescence and the solvent-dependent vanishing of the second peak has been attributed to the two singlet excited states that are responsible for the fluorescence. The first state is a locally excited state (LE) of $\pi \rightarrow \pi^{*}$ character localized on the benzene ring. The second state is a charge-transfer (CT) state, in which an electron is promoted from the electrondonating dimethylamino group towards the benzonitrile group (cf. Fig. S4 of SI). Note that none of these states is of pure LE or CT character, but rather feature mixing of different configurations. These states experience different interactions with a polar solvent and also exhibit different structural relaxation. Detailed discussion on the role of these states in dual fluorescence can be found in Ref. 76 .

DMABN example illustrates how excited states of LE and CT character can be characterized by exciton descriptors. We used several geometries from Ref. 62 to represent excited-state relaxation (see Section 3). The LE geometry is the fully-relaxed geometry optimized for the LE state. By using the sequence of the CT structures, we investigate the

effect of solvent-dependent structural relaxation by considering a twist of the two methyl groups with respect to the benzene (these structures with different twisting angles are denoted by CT0, CT45, and CT90). The fully relaxed CT state structure has a pyramidalized 
dimethylamin group (denoted CTP).
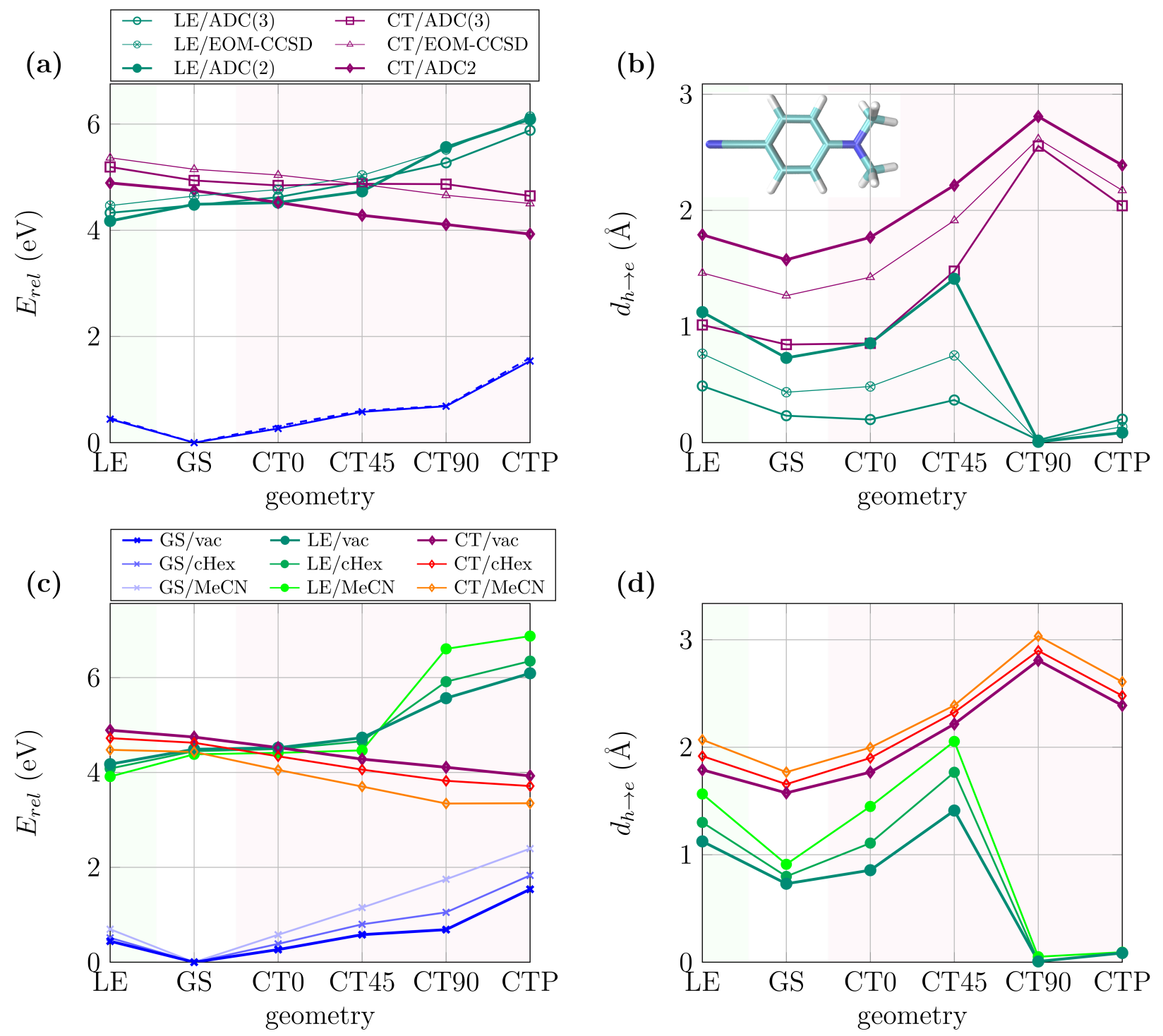

Figure 6: Lowest $\mathrm{LE}$ and $\mathrm{CT}$ states of DMABN at different geometries at the $\mathrm{ADC}(3) / \mathrm{cc}-$ pVDZ, ADC (2)/cc-pVDZ, and EOM-CCSD/cc-pVDZ levels of theory. (a) Relative excitation energies $\left(E_{r e l}, \mathrm{eV}\right)$. (b) Distances between electron and hole charge centers $\left(d_{h \rightarrow e}, \AA\right)$. Legend in (a) also applies to (b). (c) Relative energies $\left(E_{r e l}, \mathrm{eV}\right)$ of the ground (GS) and excited states (LE, CT) calculated at the $\mathrm{ADC}(2) / \mathrm{cc}-\mathrm{pVDZ}$ level of theory in combination with PCM. ${ }^{62}$ (d) Solvent effects on electron-hole separation $d_{h \rightarrow e}$. Legend in (c) also applies to $(\mathrm{d})$.

Fig. 6 presents the results for the lowest LE and CT states of DMABN at different geometries computed at the ADC(3)/cc-pVDZ, ADC(2)/cc-pVDZ, and EOM-CCSD/cc-pVDZ levels of theory. Fig. 6 (a) shows that the LE state is the lowest at the ground-state geom- 
etry for all three methods. While $\operatorname{ADC}(2)$ and $\operatorname{ADC}(3)$ values are on top of each other (4.49 $\mathrm{eV}$ and $4.47 \mathrm{eV}$, respectively), the EOM-CCSD excitation energy is slightly higher $(4.65 \mathrm{eV})$. For the CT state, the spread in excitation energies is larger: at the $\mathrm{ADC}(2)$ level, it lies only $0.26 \mathrm{eV}$ above the LE state, whereas the gap is larger $(\approx 0.5 \mathrm{eV})$ for $\mathrm{ADC}(3)$ and EOM-CCSD (the respective excitation energies are 4.94 and $5.15 \mathrm{eV}$ ). At the LE geometry (Fig. 6 (a), left side), the LE state is strongly stabilized, while the energy of the CT state is slightly blue-shifted. At the CT geometries (right side of Fig. 6 (a)), the excitation energy of the LE state slightly increases. These trends are observed at all levels of theory. At the CT90 geometry, the LE excitation energy computed by ADC(2) jumps up to $5.57 \mathrm{eV}$, in sharp contrast to $\mathrm{ADC}(3)$ and EOM-CCSD. At all levels of theory, the CT state exhibits a gradual stabilization along the twisting coordinate. The stabilization is most pronounced at the $\mathrm{ADC}(2)$ level. At the CT45 geometry, the excitation energies for the LE and CT states are almost degenerate at the $\mathrm{ADC}(3)$ and EOM-CCSD levels. Increasing the angle disrupts the conjugation between the benzene ring and the lone pair orbital at the nitrogen atom, leading to further stabilization of the CT state and destabilization of the LE state.

To rationalize the differences between the different levels of theory, we take a closer look at the excited-state properties. In the context of CT states, the vectorial electron-hole distance is the key property. For the LE state, we expect the vectorial distance between the electron and hole distribution $d_{h \rightarrow e}$ (eq. (16)) to be close to zero, since the relevant orbitals reside in the same part of the molecule, despite being delocalized (which is the case for the $\pi$ orbitals of the benzene ring). In contrast, $\mathrm{CT}$ states involve transitions between orbitals located at different parts of the molecule. Consequently, $d_{h \rightarrow e}$ should reflect the distances between the parts of the molecule involved in charge transfer. The data presented in Fig. 6 (b) shows that at the GS geometry, the LE and CT states differ in $d_{h \rightarrow e}$ by at least $0.6 \AA$ for EOM-CCSD and by more than $0.8 \AA$ for the ADC methods. At the LE optimized geometry and along the twisting coordinate, the charge separation moderately increases for the LE state and is strongly enhanced for the twisted structures (CT45 and CT90) at all levels of theory. The 
$d_{h \rightarrow e}$ values reveal major changes in the state character for the LE state, which rationalize the trends in $\mathrm{ADC}(2)$ excitation energies. For the CT45 geometry, $d_{h \rightarrow e}$ of the LE state is as large as $1.41 \AA$ which is rather typical for a CT state. At the CT90 geometry, $d_{h \rightarrow e}$ drops to almost zero. Obviously, the twist of the dimethylamin group beyond 45 degrees breaks the conjugation, and, as a consequence, the LE state localizes on the benzene ring. This effect can also be visualized using NTOs, which are presented in Fig. S4 in the SI for three geometries using the EOM-CCSD wave functions.

We note that the $d_{h \rightarrow e}$ values increase in the following sequence: $\mathrm{ADC}(3)<$ EOM-CCSD $<\operatorname{ADC}(2)$. To our knowledge, no systematic analysis of this trend has been reported so far, yet there have been a couple of studies with similar findings suggesting that $\mathrm{ADC}(2)$ tends to overestimate charge transfer character. ${ }^{62,77,78}$

To understand solvent effects on excitation energies and exciton properties, we recalculated the $d_{h \rightarrow e}$ values of the LE and CT states employing a polarizable continuum model (PCM). Fig. 6 (c) shows the results for two solvents, acetonitrile (MeCN), an example of polar solvent, and cyclo-hexane (cHex), a non-polar solvent. Fig. 6 (c) shows the relative energies for the ground and excited states with respect to the ground-state energy at the GS geometry, which is set to zero for each model. At the GS geometry, the CT state is stronger stabilized by the solvents than the LE state. At the CT optimized geometries, the ground state is strongly destabilized and its energy rises up by more than $2 \mathrm{eV}$ for the CTP geometry in MeCN. At the same time, the CT energy decreases at the CT-optimized geometries, as shown in the right hand side of Fig. 6 (c), and the stabilization is more pronounced in the polar solvent, just as expected. The LE state is slightly destabilized, with only minor influence of the environment for the smaller twisting angles. In contrast, at the CT90 and CTP geometries there are significant changes: the excitation energies steeply increase and the solvent effects become more pronounced. Comparing these trends with the changes in $d_{h \rightarrow e}$ presented in Fig. 6 (d), it is rather interesting that the effects generally follow the trend in relative energies but that the magnitude of change in $d_{h \rightarrow e}$ does not correlate linearly with 
the solvent-induced shifts. While the $d_{h \rightarrow e}$ values of the CT state show a constant shift when going from vacuum to cHex and to MeCN, irrespectively of the geometry, the LE state shows a very different trend. At the GS geometry, the shifts in $d_{h \rightarrow e}$ are almost equal to the ones of the CT state at this geometry. In contrast, the shifts are more than twice as large for the LE, CT0, and CT45 geometries. The dramatic drop in $d_{h \rightarrow e}$ at the CT90 geometry, which is attributed to the break in conjugation, is accompanied by a vanishing solvent-induced shift in $d_{h \rightarrow e}$. The solvent-dependent changes in excitation energies of the LE state at the CT90 and CTP geometries are driven by the changes in the ground-state energies at these geometries rather than by changes in the excited-state character.

In conclusion, using DMABN, we demonstrated how the $d_{h \rightarrow e}$ values of the LE and CT states are affected by electronic structure method and by solvent models. Comparing these values with the trends in relative energies revealed the origin of the sovatochromic effects. This example highlights the utility of $d_{h \rightarrow e}$ in identifying and quantifying CT character.

\subsection{Delocalized $\pi \pi^{*}$ states in hexa-thiophene}

To illustrate how exciton descriptors work in extended systems, we consider the hexa(thiophene) oligomer (6T) as an example of a large $\pi$-conjugated system in which electron-hole correlation effects become important. ${ }^{45,46,79-82}$ The key question is then how to trace and quantify these effects with simple excited-state descriptors. The theoretical description of transitions with charge-resonance character, as those giving rise to bright states in organic photovoltaic materials, is affected by the same methodological shortcomings as chargetransfer states, ${ }^{83-85}$ although the origin of the problem is obscured by the absence of permanent charge separation. ${ }^{86-88}$ Two descriptors are relevant is this case: the exciton size and the linear electron-hole correlation. While the former allows to determine the type and character of the exciton, the latter shows whether the electron-hole correlation effects are correctly described by different quantum-chemical methods. ${ }^{45,46}$

Below we consider the first singlet $\left(\mathrm{S}_{1}, 1^{1} \mathrm{~B}_{u}\right)$ and triplet $\left(\mathrm{T}_{1}, 1^{3} \mathrm{~B}_{u}\right)$ excited states. The 

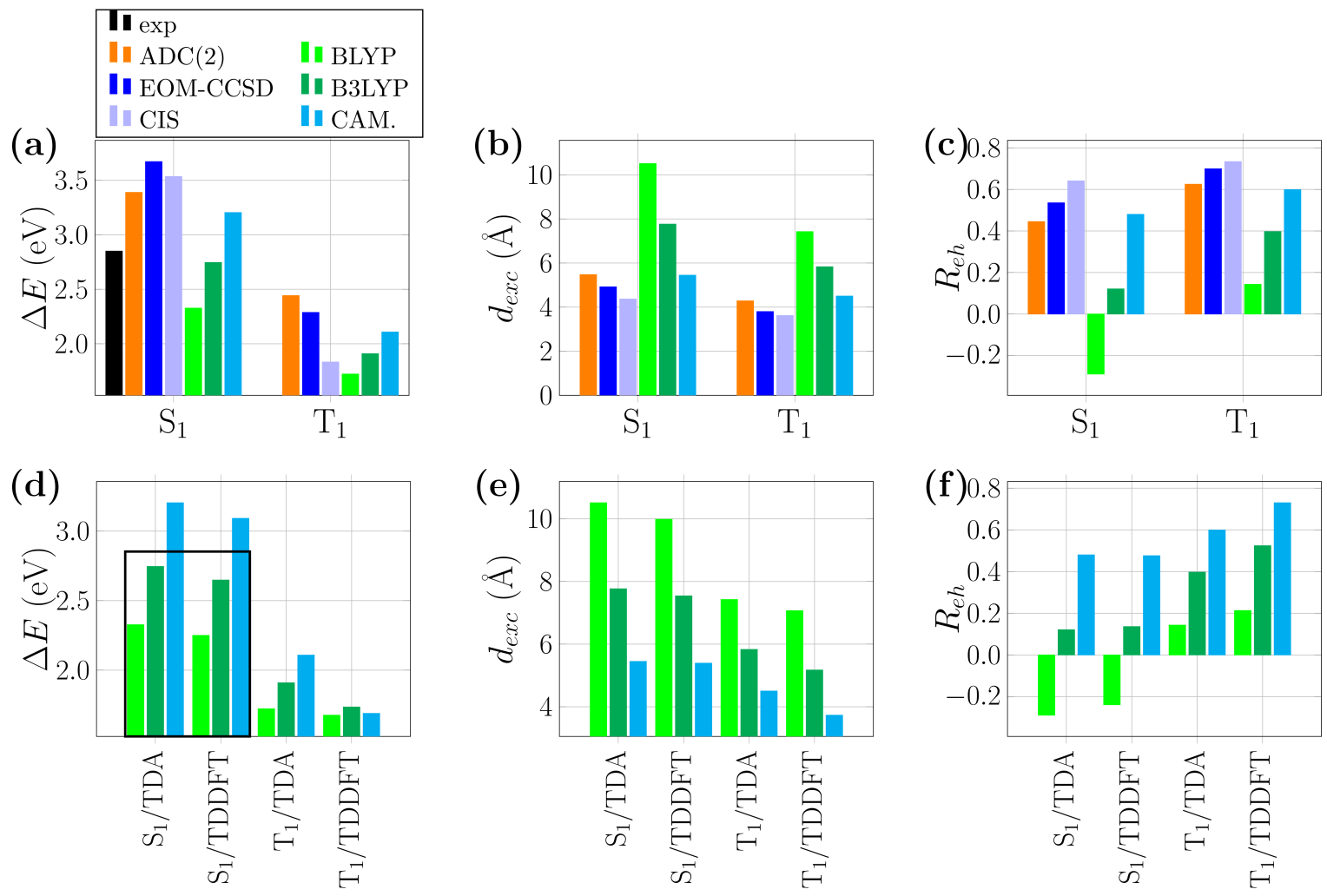

Figure 7: Excited states of hexa(thiophene). (a) and (d) Excitation energies $(\Delta E$, eV), (b) and (e) exciton sizes $\left(d_{e x c}, \AA\right)$, and (c) and (f) correlation coefficients $\left(R_{e h}\right)$ of the first singlet $\left(\mathrm{S}_{1}, 1^{1} \mathrm{~B}_{u}\right)$ and triplet $\left(\mathrm{T}_{1}, 1^{3} \mathrm{~B}_{u}\right)$ states calculated at various levels of theory employing the $\mathrm{SV}(\mathrm{P})$ basis set. The legend in (a) applies to all plots in this figure, CAM denotes CAMB3LYP. Experimental spectrum recorded in dioxane. ${ }^{89,90}$ 
$1^{1} \mathrm{~B}_{u}$ state is the lowest singlet excited state and carries large oscillator strength. Fig. 7 presents an analysis of the states in terms of excitation energies as well as exciton descriptors using $R_{e h}$ and $d_{e x c}$.

In terms of excitation energies (shown in Fig. 7(a)), $\mathrm{ADC}(2)$ and EOM-CCSD appear to significantly overestimate the excitation energy of $\mathrm{S}_{1}$, by $0.54 \mathrm{eV}$ and $0.82 \mathrm{eV}$, respectively (the experimental value is $2.85 \mathrm{eV}$ ). Let us briefly discuss several factors contributing to this rather large discrepancy. First, the experimental spectrum was recorded in dioxane, which has a refractive index of 1.42 and stabilizes excited states, thus lowering excitation energies relative to gas phase. Second, uncertainties of the geometry used in calculations may contribute to the observed discrepancies. We used geometry optimized with $\omega \mathrm{B} 97 / \mathrm{SV}(\mathrm{P})$. Reoptimizing the structure and calculating vertical excitation energies with CAM-B3LYP/cc-pVDZ yields $3.05 \mathrm{eV}$, which is a significant improvement; similar effect is expected for $\mathrm{ADC}(2)$ and EOM-CCSD. Third, using a more appropriate basis, such as aug-cc-pVTZ, should lead to additional lowering of excitation energy by $0.1-0.2 \mathrm{eV} .45,91$ While $6 \mathrm{~T}$ is too large for EOM-CCSD calculations with a triple-zeta basis, we carried out EOM-CCSD calculations with aug-cc-pVDZ basis and obtained excitation energy of $3.38 \mathrm{eV}$ (thus, reducing the error down to $0.53 \mathrm{eV}$ ). Fourth, the effect of higher excitations is important. Although the bright $\mathrm{B}_{u}$ state is a singly excited state, doubly and triply excited determinants are important for dynamical correlation. States with large charge-resonance character (such as singlet $\pi \pi^{*}$ states in conjugated systems) are known to require accurate account of dynamic correlation, e.g., for these states the errors of EOM-CCSD are often close to $0.3 \mathrm{eV}$ and systematic, leading to overestimation of excitation energies (cf. Ref. 91). The importance of dynamic correlation is clearly seen by comparing CIS to ADC(2) and EOMCCSD: Including doubly excited determinants yields better excitation energies. Finally, we note that for unambiguous comparison between the experimental absorption spectra and theoretical results one needs to compute the vibrational envelope (Franck-Condon factors). Such calculations are costly and are outside of the scope of this work. Previous studies have 
shown that the absorption band maxima in polyatomic molecules is often closer to adiabatic (00) transitions rather than to vertical energy difference. ${ }^{92-94}$ Thus, the account of structural relaxation can lead to additional red shift in the computed energies.

Among TDDFT methods, the B3LYP shows the best agreement with experimental absorption maximum (error of $-0.10 \mathrm{eV}$ ), CAM-B3LYP result is blue-shifted by $0.35 \mathrm{eV}$, and BLYP underestimates the excitation energy by $-0.52 \mathrm{eV}$. We note that blue shift observed with CAM-B3LYP is consistent with the behavior of wave function methods, whereas seemingly better agreement of BLYP and B3LYP is likely an artifact.

For the $\mathrm{T}_{1}$ state $\left({ }^{3} \mathrm{~B}_{u}\right)$, EOM-CCSD and $\mathrm{ADC}(2)$ yield very similar results: The EOMCCSD excitation energy is $2.29 \mathrm{eV}$, which is only $-0.15 \mathrm{eV}$ below the $\mathrm{ADC}(2)$ value of $2.44 \mathrm{eV}$. In contrast, TDDFT (and CIS) methods yield much smaller values clustered around $1.7 \mathrm{eV}$.

Figs. 7(b) and (c) show exciton descriptors, revealing a large spread in exciton sizes and correlation coefficients for the two states. For the $\mathrm{S}_{1}$ state, EOM-CCSD exciton size is $5 \AA$, which is similar to the CAM-B3LYP value. B3LYP and BLYP lead to much larger values of $8 \AA$ and $10 \AA$, respectively. Comparing these values to the size of the molecule $(22.1 \AA)$ and taking into account that we compute the root-mean-square value of the electronhole distribution, we infer that EOM-CCSD, $\mathrm{ADC}(2)$, and CAM-B3LYP predict a spatially confined electron-hole pair, whereas BLYP and B3LYP yield fully delocalized distribution of electron and hole. The exciton size in $T_{1}$ is much smaller for all methods, which can be attributed to the absence of exchange repulsion.

The electron-hole correlation coefficients (Fig. 7(c)) illuminate the observed wide spread in exciton sizes for the $\mathrm{S}_{1}$ state: While for the ab initio methods and CAM-B3LYP $R_{e h}$ is positive with values above 0.44, BLYP exhibits a negative electron-hole correlation (-0.29), and B3LYP shows small positive value $(+0.12)$. A negative value in $R_{e h}$ corresponds to a dynamical avoidance of the electron and hole in space, which is physically incorrect, because in molecules they should attract each other. A related study on tetra(thiophene) has shown 
that the excitation energies of the first bright excited state strongly depend on the amount of non-local exact exchange and that spurious charge-transfer states appear for functionals without or with low percentage of non-local orbital exchange. ${ }^{95}$ The same phenomenon is at play in $\pi$-conjugated systems and is related to methodological issues in the description of excitonic properties. ${ }^{46}$ Our results therefore show yet another example of misleading error cancellation, illustrating that seemingly accurate excitation energies produced by B3LYP correspond to completely wrong description of the exciton.

In the triplet state, all methods yield $R_{e h}$ which is larger compared to the $\mathrm{S}_{1}$ values. The increase in electron-hole correlation can be rationalized in terms of exchange interaction: While electron and hole experience a short-range repulsion in the case of singlet excitons, in triplet excitons Pauli hole screens the Coulomb hole thus allowing the hole and particle to co-localize in space. This phenomenon, clearly seen in $d_{e x c}$ and $R_{e h}$, is a short-range effect and largely determined by the response of the Hartree potential. Consequently, it is well captured by TDDFT with all functionals.

In the context of a debate of the applicability of the TDA approximation in extended $\pi$-systems, Fig. $7(\mathrm{~d})$ compares the $\mathrm{S}_{1}$ excitation energies computed with and without TDA with BLYP, B3LYP, and CAM-B3LYP. The black box represents the experimental value of $2.85 \mathrm{eV} .{ }^{89,90}$ While for the BLYP and B3LYP functionals we observe smaller errors when employing TDA, for CAM-B3LYP the full TDDFT seems to perform better $(+0.24$ eV versus $+0.35 \mathrm{eV})$.

\subsection{Doubly excited states in octatetraene}

All-trans polyenes, prototypical linear conjugated systems, play key roles in many biologically relevant systems, e.g., in carotenoids. Despite their simple structure, excited states of polyenes are challenging for theory, due to low-lying doubly excited configurations. ${ }^{51,96,97} \mathrm{~A}$ plethora of studies have investigated different aspects of the excited states of polyenes. ${ }^{68,96-104}$ We use this system to take a closer look on how to identify doubly, or, more generally, multi- 
ply excited states, and to compare excited states and their properties computed with different methods.

In many biologically relevant applications, excited-state calculations are only feasible using very efficient but approximate TDDFT approach. An intense search for an appropriate exchange-correlation functional revealed that BLYP yields the experimentally observed state order, i.e., that the dark $A_{g}$ state is the lowest excited state for all compounds larger than hexatriene. For this reason, we included the BLYP functional in the benchmark set, along with its hybrid counterpart, B3LYP, and a long-range corrected functional, CAM-B3LYP. It is well known that linear-response TDDFT usually performs poorly for doubly excited states. It would be interesting to compare these methods with alternative approaches, e.g. dressed TDDFT or spin-flip TDDFT, ${ }^{105,106}$ cf. e.g. Ref. 13 and references therein. However, this is beyond the scope of the present work.

The excited states are classified in terms of their irreducible representation as $\mathrm{A}_{g}, \mathrm{~A}_{u}$, $\mathrm{B}_{g}$, and $\mathrm{B}_{u}$ and marked with + or - depending on their character, i.e., + denotes ionic resonance structures and - denotes to neutral (covalent) ones (cf. Ref. 104 and references therein).

Fig. 8 presents the results for the $2^{1} \mathrm{~A}_{g}^{-}, 1^{1} \mathrm{~B}_{u}^{+}$, and $1^{1} \mathrm{~B}_{u}^{-}$excited states of octatetraene. Fig. 8(a) shows a large spread in the excitation energies computed by different methods. $\mathrm{ADC}(3)$ shows the best agreement with the experimental data, ${ }^{107}$ with deviations of +0.36 $\mathrm{eV}$ and $+0.27 \mathrm{eV}$ for $2^{1} \mathrm{~A}_{g}^{-}$and for $1^{1} \mathrm{~B}_{u}^{+}$, respectively. $\mathrm{ADC}(3)$ is the only ab initio method that reproduces the experimental state ordering. $\mathrm{ADC}(2)$ and EOM-CCSD show substantial errors for the $2^{1} \mathrm{~A}_{g}^{-}$state $(+2.39 \mathrm{eV}$ for $\mathrm{ADC}(2)$ and $+2.54 \mathrm{eV}$ for EOM-CCSD). For the $1^{1} \mathrm{~B}_{u}^{+}$state, the errors are much smaller: $+0.24 \mathrm{eV}$ and $+0.62 \mathrm{eV}$ for $\mathrm{ADC}(2)$ and EOMCCSD, respectively. Increasing the basis set up to aug-cc-pVTZ lowers the EOM-CCSD excitation energies by about $0.1 \mathrm{eV}$ yielding 6.055 and $4.896 \mathrm{eV}$ for the $2^{1} \mathrm{~A}_{g}^{-}$and $1^{1} \mathrm{~B}_{u}^{+}$ states, respectively. Thus, even with a larger basis EOM-CCSD still places the dark $A_{g}$ state above the bright $B_{u}$ state vertically. 

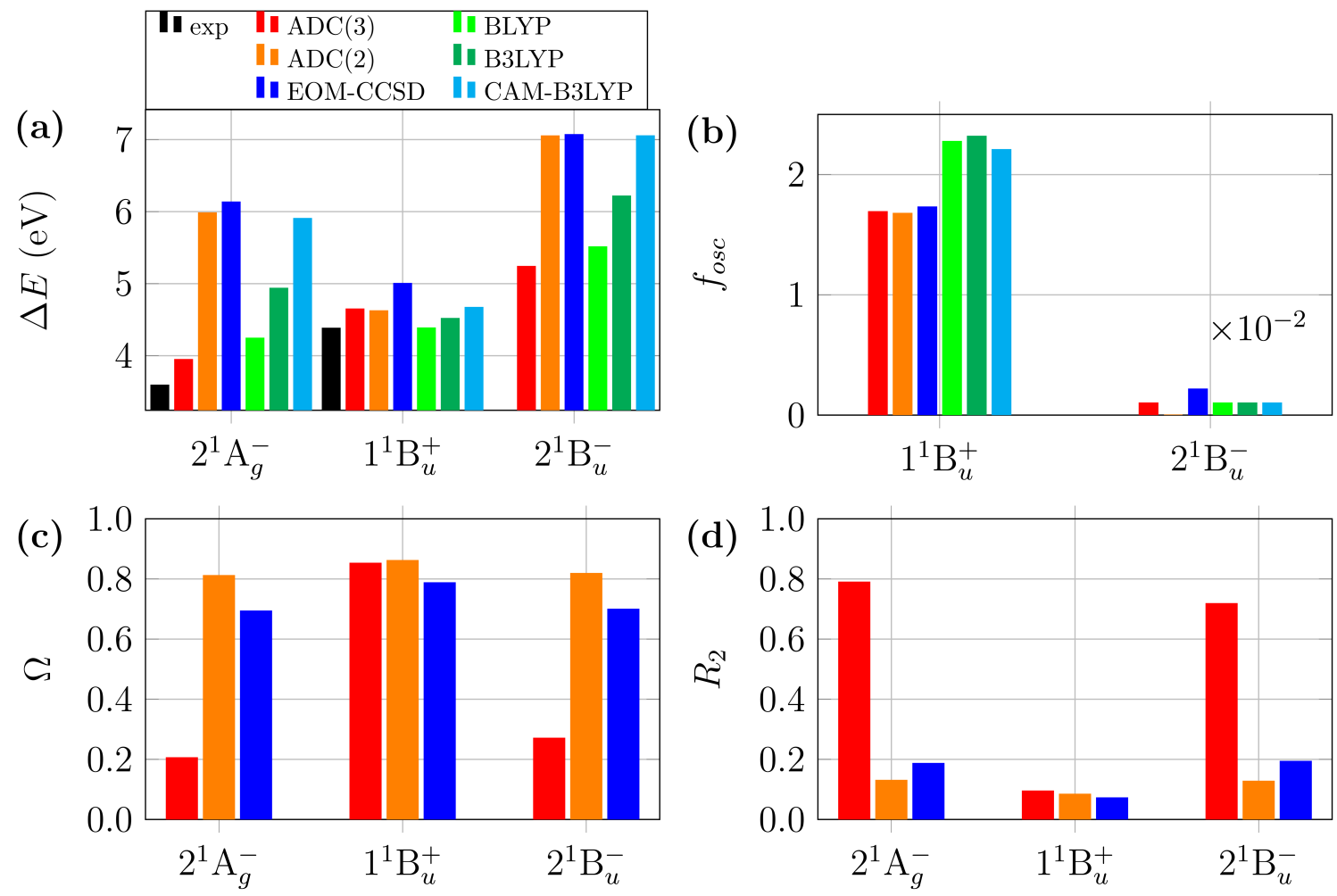

Figure 8: Excited states of octatetraene. (a) Excitation energies $(\Delta E, \mathrm{eV}),(\mathrm{b})$ oscillator strengths $\left(f_{\text {osc }}\right),(\mathrm{c}) \Omega$ values $(\Omega)$, and $(\mathrm{d})$ squared doubles amplitudes $\left(R_{2}\right)$ of the $2^{1} \mathrm{~A}_{g}^{-}, 1^{1} \mathrm{~B}_{u}^{+}$, and $2^{1} \mathrm{~B}_{u}^{-}$states calculated with $\mathrm{ADC}(2)$, ADC(3), EOM-CCSD, BLYP/TDA, B3LYP/TDA, and CAM-B3LYP/TDA employing the cc-pVTZ basis set. The legend in (a) applies to all diagrams. Experimental data are from Ref. 107.

Exciton analysis allows us to rationalize these large discrepancies between the methods. The key quantity here is the amount of single and double (or multiple) excitation character. Fig. 8(c) and (d) shows two measures, the squared norm of the exciton wave function $\Omega$ and the squared value of the doubles amplitudes $R_{2}$ in the excited states. An advantage of using $\Omega$ is that the respective values are orbital invariant and well-defined independent from the computational protocol, which affords a more precise comparison than using $R_{2}$.

The $\mathrm{ADC}(3)$ values clearly indicate a predominant double excitation character in the $2^{1} \mathrm{~A}_{g}^{-}$and $2^{1} \mathrm{~B}_{u}^{-}$excited states. $\mathrm{ADC}(2)$ fails to capture this effect even qualitatively: all three states have $\Omega$ values larger than 0.8 , completely missing out differences in state character and confirming the results of Ref. 108. Despite relatively large errors in excitation energies, the EOM-CCSD $\Omega$ values indicate that EOM-CCSD captures a small difference in excitation 
character, in accordance with the trends observed at the $\mathrm{ADC}(3)$ level. The $R_{2}$ values follow similar trend as the $\Omega$ values.

Given the differences in state characters, it is not surprising that the $\operatorname{ADC}(3)$ method, which describes doubly excited determinants at first order in perturbation theory, performs much better than $\mathrm{ADC}(2)$, which describes these determinants only at zeroth order. In the EOM-CCSD ansatz, the double excitations are included explicitly, but their primary role is to describe dynamical correlation for singly excited configurations. Thus, when excited states have predominantly doubly excited character, there are no higher-level configurations to correlate these states. An explicit inclusion of double excitations in EOM-CCSD is responsible for its superiority relative to $\operatorname{ADC}(2)$. Further discussion on doubly excited states can be found in Ref. 97.

Let us now discuss the performance of TDDFT, cf. Fig. 8(a). At the first glance, BLYP shows the best agreement with the experimental data and the $\mathrm{ADC}(3)$ results. While CAM-B3LYP closely follows $\mathrm{ADC}(2)$ and EOM-CCSD, the excitation energies obtained with B3LYP are almost exactly in between BLYP and CAM-B3LYP for all three states. As TDDFT only includes singly excited determinants, $\Omega=1$ for all TDDFT states, meaning that doubly (or multiply) excited character cannot be described. ${ }^{99}$ To rationalize the differences and similarities of the computed excited-state wave functions, Fig. 9 shows exciton properties such as exciton sizes and correlation coefficients. We note that these descriptors only characterize the part of the excited state that is described by single electron transitions, because they are based on the one-particle transition density matrix. For example, predominantly doubly excited state $2^{1} \mathrm{~A}_{g}^{-}$has $\Omega=0.2$ at the $\mathrm{ADC}(3)$ level. This means that only $20 \%$ of the excited state can be described within the exciton model. Consequently, exciton sizes and correlation coefficients in Fig. 9 are not very meaningful for the $2^{1} \mathrm{~A}_{g}^{-}$and $2^{1} \mathrm{~B}_{u}^{-}$states.

For the predominantly singly excited state $1^{1} \mathrm{~B}_{u}^{+}$, all wave function based methods are in good agreement with an exciton size of about $4 \AA$ and a slightly positive correlation coefficient of +0.1 . Among TDDFT methods, CAM-B3LYP shows the best agreement with 

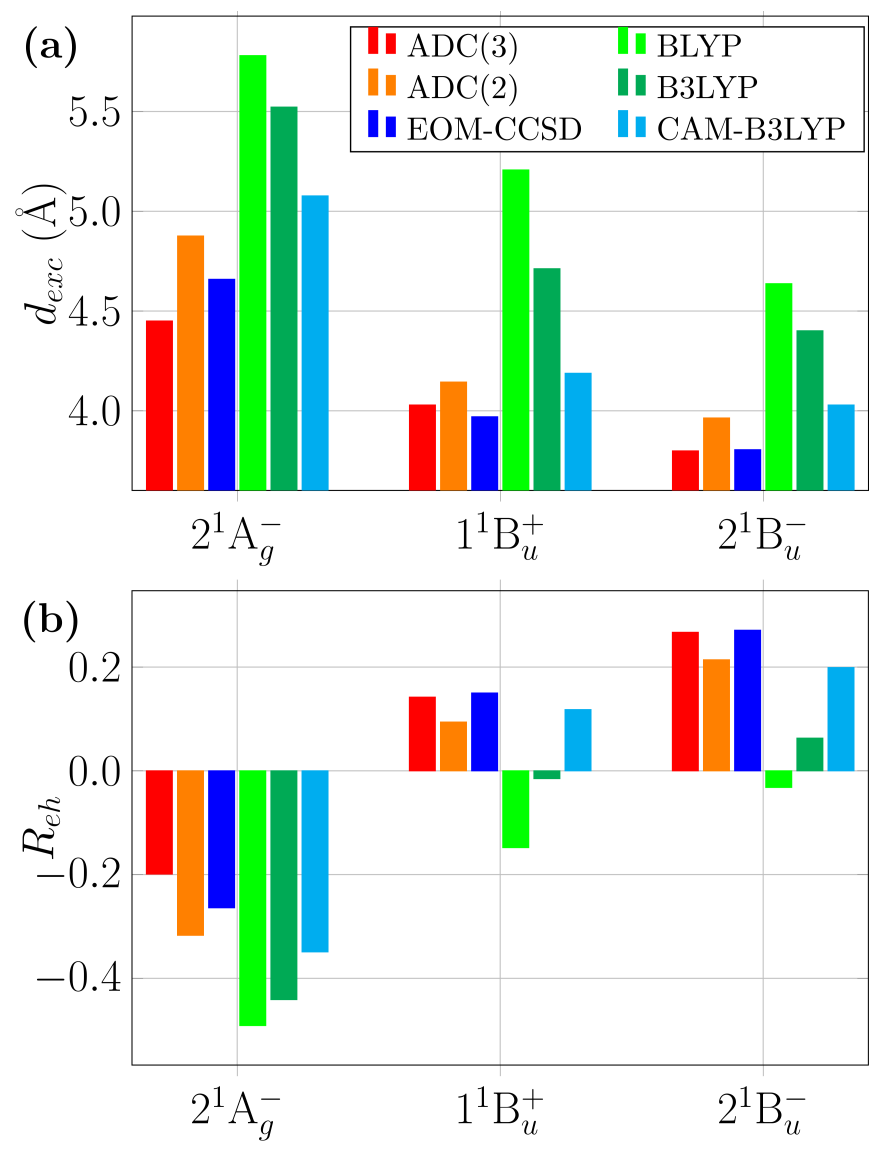

Figure 9: Excited states of octatetraene. (a) Exciton sizes $\left(d_{\text {exc }}, \AA\right)$ and (b) correlation coefficients $\left(R_{e h}\right)$ of the $2^{1} \mathrm{~A}_{g}^{-}, 1^{1} \mathrm{~B}_{u}^{+}$, and $2^{1} \mathrm{~B}_{u}^{-}$excited states calculated with $\mathrm{ADC}(2), \operatorname{ADC}(3)$, EOM-CCSD, BLYP/TDA, B3LYP/TDA, and CAM-B3LYP/TDA employing the cc-pVTZ basis set. The legend in (a) applies to both diagrams.

these values. In contrast, for BLYP, $d_{e x c}$ is much larger $(5.21 \AA)$ and $R_{e h}$ is negative $(-0.148)$.

Other states (those with substantial single excitation character) are described consistently by the wave function based methods suggesting the major differences in excitation energies originates from the doubly or multiply excited determinants. It would be interesting to further characterize this phenomenon, however, more advanced techniques need to be employed, cf. Ref. 109, which is beyond the scope of this work. Using ADC(3) as a reference, we observe the best agreement in exciton properties for EOM-CCSD, while ADC(2) shows the largest discrepancies for the $2^{1} \mathrm{~A}_{g}^{-}$state. In contrast to the good agreement in excitation energies, the exciton sizes obtained with BLYP deviate the most from the ADC(3) values. We observe a systematic decrease in errors against $\mathrm{ADC}(3)$ when going from BLYP 
to B3LYP to CAM-B3LYP, which effectively corresponds to an increase of non-local orbital exchange. ${ }^{13,46}$

Fig. 9(b) plots electron-hole correlation coefficient $R_{e h}$, showing a qualitative agreement among all wave function based methods. We observe an opposite trend in terms of deviation from the $\mathrm{ADC}(3)$ reference: When the correlation is smaller, then the exciton size increases with respect to the reference. While for the predominantly doubly excited state, the electronhole correlation is negative, i.e., the electron and hole dynamically avoid each other in space, the positive $R_{e h}$ values for the second and third state are slightly positive.

In summary, we characterized the low-lying excited states of octatetraene in terms of double excitation character and exciton properties, confirming the findings of Ref. 97. Interestingly, in the doubly excited $2^{1} \mathrm{~A}_{g}^{-}$state, there is negative correlation between electron and hole. CAM-B3LYP is the only $x c$-functional tested here that agrees with the ab initio methods when it comes to exciton properties, while its performance is rather poor in terms of excitation energies in contrast to, e.g. BLYP. Ref. 99 has pointed out that excited states in polyenes require a more accurate treatment of electron correlation than provided by simple gradient-corrected functionals. Our results confirm this conclusion, suggesting that longrange corrections are unlikely to compensate the errors for polyenes and related molecules inherited from the simpler $x c$-functionals.

\subsection{Magnesium(II)porphyrin}

Porphyrins are a very important class of molecules present in active centers of biologically and physiologically relevant molecules. Magnesium(II)porphyrin is a model for chlorophyll, which has been an object of intense studies ever since its discovery in 1940. Using this molecule as a representative of a large molecular class with rich photochemistry, we illustrate how benchmarking and interpretation of the excited states can benefit from exciton analysis.

The excited-state absorption spectrum of magnesium(II)porphyrin ( $\mathrm{MgP})$ is rationalized using a four-orbital model by Gouterman, ${ }^{110-112}$ which explains the origin of the $Q$ and $B$ 
bands. While drawing a full picture of all relevant excited states is beyond the scope of this work, we focus on the first bright excited state related to the low-intensity $Q$ band and on the interpretation of the four doubly degenerate, bright states in comparison to Gouterman's model.

Table 2: The first excited $Q$ state of Magnesium(II)porphyrin calculated at various levels of theory employing Ahlrichs' SV $(\mathrm{P})^{59}$ basis set if not stated otherwise.

\begin{tabular}{|c|c|c|c|c|c|c|c|}
\hline method & $\Delta \mathrm{E}(\mathrm{eV})$ & $\mathrm{f}$ & $\Omega$ & $d_{e x c}$ & $\sigma_{h}$ & $\sigma_{e}$ & $R_{e h}$ \\
\hline exp. & $2.07^{a}$ & & & & & & \\
\hline $\operatorname{ADC}(3)$ & 1.996 & 0.008 & 0.728 & 4.58 & 3.50 & 3.65 & 0.179 \\
\hline $\operatorname{ADC}(2)$ & 2.382 & 0.007 & 0.757 & 4.64 & 3.38 & 3.67 & 0.169 \\
\hline $\mathrm{EOM}^{-C C S D}{ }^{b}$ & 2.344 & 0.001 & 0.759 & 4.58 & 3.45 & 3.65 & 0.167 \\
\hline $\mathrm{EOM}^{-\mathrm{CCSD}^{b, c}}$ & 2.345 & 0.007 & 0.736 & 4.62 & 3.46 & 3.64 & 0.158 \\
\hline $\mathrm{EOM}^{-\mathrm{CCSD}^{b, d}}$ & 2.302 & 0.007 & 0.741 & 4.65 & 3.49 & 3.67 & 0.158 \\
\hline BLYP & 2.265 & 0 & 1 & 5.17 & 3.46 & 3.70 & -0.048 \\
\hline B3LYP & 2.388 & 0.001 & 1 & 5.03 & 3.47 & 3.68 & 0.012 \\
\hline CAM-B3LYP & 2.423 & 0.004 & 1 & 4.81 & 3.48 & 3.65 & 0.090 \\
\hline CIS & 2.432 & 0.038 & 1 & 4.68 & 3.55 & 3.67 & 0.160 \\
\hline
\end{tabular}

Let us begin by discussing the benchmark data set for the first bright excited state shown in Table 2. Comparing the excitation energies obtained with the different computational methods with the experimental value of $2.07 \mathrm{eV},{ }^{110}$ the $\mathrm{ADC}(3)$ method shows the best agreement (excitation energy of $1.996 \mathrm{eV}$ ). The second smallest error obtained with BLYP is already substantially larger, with excitation energy of $2.265 \mathrm{eV}$. All other methods exhibit a uniform blue shift of about $0.3 \mathrm{eV}$ (or even $+0.4 \mathrm{eV}$ for CAM-B3LYP). It is worth noting that EOM-CCSD and $\mathrm{ADC}(2)$ excitation energies are very close $(2.344 \mathrm{eV}$ and $2.382 \mathrm{eV}$, respectively) and that B3LYP is almost on top of these values $(2.388 \mathrm{eV})$. For EOM-CCSD, we also investigated the effects of the basis set. While the value obtained with Dunning's double- $\zeta$ basis is within $0.001 \mathrm{eV}$ from the value obtained with Ahlrichs' $\mathrm{SV}(\mathrm{P})$ basis, using triple- $\zeta$ basis leads to an improved agreement with the experiment $(2.30 \mathrm{eV})$.

Despite the discrepancies in the excitation energies, the exciton properties of the first 
excited state are consistent among the wave function-based methods (ADC(3), ADC(2), and EOM-CCSD). This is especially true when comparing $\mathrm{ADC}(3)$ and EOM-CCSD. The $\Omega$ values indicate a similar amount of single excitation character $(0.75)$ and other exciton descriptors are very similar. The $\mathrm{ADC}(2)$ descriptors show larger differences, but are nevertheless in a good overall agreement.

Considering differences between the tested TD-DFT methods, the most interesting trend can be attributed to a gradual increase of exact exchange. In the BLYP functional no nonlocal orbital exchange is included, while a constant fraction of $21 \%$ is included for B3LYP, and a range-separation function admixes non-local orbital exchange between $19 \%$ and $65 \%$ in CAM-B3LYP. While the best results for the excitation energy appears to be obtained with BLYP, it is noteworthy that the electron-hole correlation is negative $\left(R_{e h}=-0.048\right)$. As in the case of hexa(thiophene), $\mathrm{ADC}(3)$ results suggest that electron-hole correlation should be positive. Increasing the amount of exact exchange, which corresponds to introducing the electron-hole attraction in the framework of TDDFT, ${ }^{13,46}$ improves the description of the electron-hole correlation yielding positive values of $R_{e h}$. But while $R_{e h}$ value is improved when going from BLYP to B3LYP to CAM-B3LYP, the excitation energies become worse, approaching the typical error of CIS.

As the next step, we analyze the first four doubly degenerate $\pi \pi^{*}$ states of MgP employing TD-DFT and a triple- $\zeta$ basis set and attempt to reconstruct Gouterman's model. The TDDFT results, experimental data, ${ }^{110}$ and the $\mathrm{ADC}(2) / \mathrm{SV}(\mathrm{P})$ reference values are presented in Table 3 (we report the results for one state from each degenerate pair, as the second state has identical properties). Following the trends for $\mathrm{S}_{1}$, the results illustrate how difficult it is to describe excited states of large conjugated systems highlighting a true dilemma. While BLYP delivers reasonable excitation energies, the negative correlation between hole and electron reflects a qualitatively wrong description of the corresponding exciton. Adding exact exchange (as, e.g., in CAM-B3LYP) improves exciton properties yielding the expected positive electron-hole correlation as in $\operatorname{ADC}(2)$, but at the price of increased errors in excitation 
Table 3: Characterization of the four doubly degenerate, bright excited states of $\mathrm{Mg}(\mathrm{II})$ porphyrin in terms of excitation energies $\left(\Delta \mathrm{E}\right.$, eV), oscillator strengths $\left(f_{\text {osc }}\right)$, exciton sizes $\left(d_{e x c}(\AA)\right)$, hole and electron sizes $\left(\sigma_{h}(\AA), \sigma_{e}(\AA)\right)$, and correlation coefficients $\left(R_{e h}\right)$ calculated by CIS and TD-DFT with the BLYP, B3LYP, and CAM-B3LYP functionals employing Ahlrichs' TZVP basis set.

\begin{tabular}{llllllll} 
method & state & $\Delta \mathrm{E}(\mathrm{eV})$ & $f_{\text {osc }}$ & $d_{\text {exc }}(\AA)$ & $\sigma_{h}(\AA)$ & $\sigma_{e}(\AA)$ & $R_{e h}$ \\
\hline exp. & $Q$ & $2.07^{a}$ & & & & & \\
BLYP & $\mathrm{S}_{1}$ & 2.271 & 0 & 5.20 & 3.47 & 3.72 & -0.046 \\
B3LYP & $\mathrm{S}_{1}$ & 2.393 & 0 & 5.05 & 3.48 & 3.70 & 0.011 \\
CAM-B3LYP & $\mathrm{S}_{1}$ & 2.430 & 0.001 & 4.83 & 3.48 & 3.66 & 0.088 \\
ADC $(2)^{b}$ & $1^{1} \mathrm{~B}_{2 u}$ & 2.382 & 0.007 & 4.64 & 3.38 & 3.67 & 0.136 \\
CIS & $\mathrm{S}_{1}$ & 2.465 & 0.020 & 4.71 & 3.56 & 3.68 & 0.155 \\
\hline exp. & $B$ & $3.05^{a}$ & & & & & \\
BLYP & $\mathrm{S}_{3}$ & 3.156 & 0.016 & 5.55 & 3.77 & 3.71 & -0.101 \\
B3LYP & $\mathrm{S}_{4}$ & 3.676 & 0.110 & 5.14 & 3.70 & 3.70 & 0.038 \\
CAM-B3LYP & $\mathrm{S}_{3}$ & 4.098 & 1.123 & 4.65 & 3.43 & 3.79 & 0.172 \\
ADC $(2)^{b}$ & $2^{1} \mathrm{~B}_{2 u}$ & 3.487 & 1.357 & 4.88 & 3.40 & 3.76 & 0.075 \\
CIS & $\mathrm{S}_{3}$ & 4.578 & 2.698 & 4.88 & 3.38 & 3.80 & 0.079 \\
\hline exp. & $N$ & $3.97^{a}$ & & & & & \\
BLYP & $\mathrm{S}_{10}$ & 3.441 & 0.064 & 5.47 & 3.75 & 3.77 & -0.059 \\
B3LYP & $\mathrm{S}_{8}$ & 3.947 & 0.593 & 5.04 & 3.45 & 3.82 & 0.042 \\
CAM-B3LYP & $\mathrm{S}_{7}$ & 4.402 & 0.992 & 4.93 & 3.60 & 3.71 & 0.092 \\
ADC $(2)^{b}$ & $3^{1} \mathrm{~B}_{2 u}$ & 3.993 & 0.007 & 4.89 & 3.62 & 3.68 & 0.105 \\
CIS & $\mathrm{S}_{7}$ & 5.274 & 0.323 & 4.44 & 3.79 & 3.80 & 0.314 \\
\hline BLYP & $\mathrm{S}_{15}$ & 3.973 & 1.583 & 5.60 & 3.72 & 4.00 & -0.052 \\
B3LYP & $\mathrm{S}_{13}$ & 4.340 & 1.722 & 5.63 & 4.05 & 3.79 & -0.031 \\
CAM-B3LYP & $\mathrm{S}_{12}$ & 4.994 & 0.798 & 5.28 & 4.07 & 3.67 & 0.072 \\
ADC $(2)^{b}$ & $4^{1} \mathrm{~B}_{2 u}$ & 4.625 & 0.478 & 5.26 & 4.06 & 3.66 & 0.075 \\
CIS & $\mathrm{S}_{16}$ & 6.235 & 0.729 & 4.92 & 3.94 & 3.66 & 0.163 \\
\hline
\end{tabular}

${ }^{a}$ From Ref. 110. ${ }^{b}$ Symmetric structure, SV(P) basis. 
energies, which are overestimated by $0.36-1.00 \mathrm{eV}$.
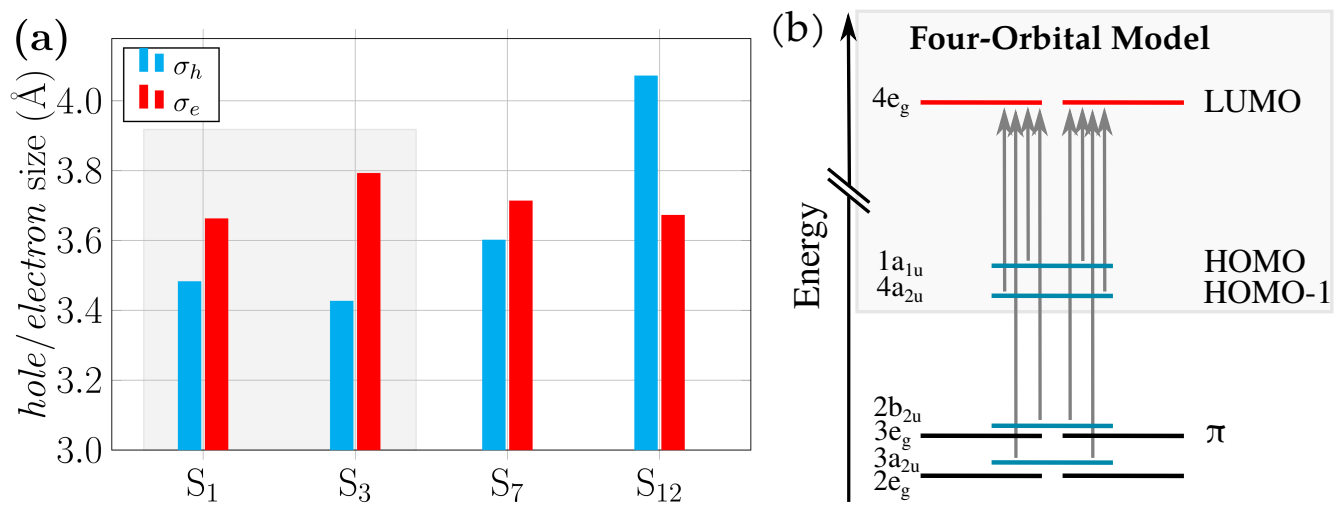

Figure 10: Gouterman's model: (a) hole and electron sizes $\left(\sigma_{h}, \sigma_{e}, \AA\right)$ of four doubly degenerate excited states calculated at the CAM-B3LYP/TZVP level of theory, (b) Sketch of orbitals and transitions involved in the absorption spectrum of $\mathrm{MgP}$ including the classic Four-Orbital model (grey), adopted from Ref. 113.

Fig. 10 (a) visualizes the hole and electron sizes of the four bright states computed with CAM-B3LYP/TZVP. While the electron sizes (red) are nearly the same (with a mean value of $3.71 \pm 0.06 \AA$, the hole sizes (blue) show larger variation around the mean value of $3.65 \pm 0.29 \AA$. Nearly constant $\sigma_{e}$ values are consistent with the Four-Orbital model of Gouterman in which the two degenerate LUMOs act as final orbitals in all bright states. ${ }^{110}$ At the same time, the variations in $\sigma_{h}$ indicate the different types of initial orbitals. An inspection of the NTOs shown in Fig. S8 in the Supplementary Information confirms these observations: All final (electron) orbitals have the same shape with only minor differences in amplitudes. $\mathrm{S}_{1}$ and $\mathrm{S}_{3}$ contain admixture of the same initial orbitals $(50 \%$ and $36 \%$, respectively), in agreement with the Four-Orbital model. For the $\mathrm{S}_{12}$ state, the hole orbitals are localized on the porphyrin ring with amplitudes in the largest possible distance to each other which results in the largest $\sigma_{h}$. The metal center does not participate in the NTO pairs of these states.

Summarizing the $\mathrm{MgP}$ results, $\mathrm{ADC}(3)$ yields the best agreement with the experimental value of the $\mathrm{S}_{1}$ excitation energy. The EOM-CCSD excitation energy is blue-shifted by $\sim 0.25 \mathrm{eV}$, but the respective exciton properties are in excellent agreement with $\mathrm{ADC}(3)$. 
TD-DFT results depend strongly on the amount of exact exchange. Increased fraction of exact exchange improves exciton description, but leads to larger errors in excitation energies. The NTO analysis of the four bright states responsible for the visible MgP spectrum confirm the validity of Gouterman's model.

\section{Summary and conclusion}

This work presented a new strategy for benchmarking excited-state calculations that goes beyond excitation energies by exploiting exciton analyses. By using a comprehensive set of examples ranging from small to large molecules with different types of excited states, we illustrated the utility of exciton analyses in benchmarking. The study considered wave function methods, EOM-CCSD, $\mathrm{ADC}(2)$, and $\mathrm{ADC}(3)$, as well as TDDFT in combination with three functionals (BLYP, B3LYP, and CAM-B3LYP).

We illustrated that electron sizes provide a convenient tool for differentiating between Rydberg and valence excited states, while electron-hole distance allows one to quantify the amount of charge transfer. Electron-hole correlation illuminates the nature of excitons and reveals important methodological aspects, such as qualitatively different description of singlets and triplets by TDDFT. The analysis of exciton properties in large conjugated systems highlights the limitations of energy-based benchmarking: we show that for TDDFT methods the best agreement in terms of excitation energies corresponds to rather poor (and even qualitatively incorrect) exciton description, while improving the exciton description by increasing the fraction of exact exchange leads to increased errors in excitation energies.

Exciton properties facilitate the assignment of state characters and also deliver important information about electron-hole correlation effects, thus establishing a new criterion for benchmarking. Importantly, the analysis based on density matrix and exciton properties enables unambiguous comparisons of different many-body wave functions and between wave function-based and DFT methods. While the focus of this work is on comparisons between 
single-reference methods, we note that recently such analysis of exciton properties has been implemented ${ }^{114}$ within the framework of multireference methods, CASSCF and CASPT2, which will facilitate future comparisons between single- and multi-reference methods.

\section{Acknowledgement}

The authors thank Dr. Jan-Michael Mewes for fruitful discussion and sharing data about excited states of DMABN. S.A.M. acknowledges funding of the Heidelberg Graduate School of Mathematical and Computational Methods for the Sciences. A.I.K. acknowledges support by the U.S. National Science Foundation (No. CHE-1566428).

\section{Supporting Information Available}

Exciton and hole sizes of formaldehyde calculated with $\mathrm{ADC}(3)$. Comparison between $\mathrm{ADC}(3), \mathrm{ADC}(2)$, and EOM-CCSD descriptions of eight excited state formaldehyde. Isovalues and their influence on orbital representations. NTOs of DMABN at EOM-CCSD

level. Singlet excitation energies of oligothiophenes at various levels of theory. NTOs of hexa(thiophene) at $\mathrm{ADC}(2)$ and TDDFT/BLYP levels. MgP excited states at various levels of theory using SV, SV(P), and TZVP basis sets. NTOs of MgP at TDDFT/TDA/CAMB3LYP level. Raw data of all plots. Cartesian coordinates of all molecules. This material is available free of charge via the Internet at http://pubs.acs.org/. 


\section{References}

(1) Helgaker, T.; Jørgensen, P.; Olsen, J. Molecular electronic structure theory; Wiley \& Sons, 2000.

(2) Dreuw, A. Quantum Chemical Methods for the Investigation of Photoinitiated Processes in Biological Systems: Theory and Applications. ChemPhysChem 2006, 7, $2259-2274$.

(3) Koch, H.; Christiansen, O.; Jørgensen, P.; Olsen, J. Excitation Energies of BH, $\mathrm{CH}_{2}$, and Ne in Full Configuration Interaction and the Hierarchy CCS, CC2, CCSD, and CC3 of Coupled Cluster Models. Chem. Phys. Lett. 1995, 244, 75-82.

(4) Schreiber, M.; Silva-Junior, M. R.; Sauer, S. P. A.; Thiel, W. Benchmarks for Electronically Excited States: CASPT2, CC2, CCSD, and CC3. J. Chem. Phys. 2008, 128, 134110.

(5) Jacquemin, D.; Wathelet, V.; Perpte, E. A.; Adamo, C. Extensive TD-DFT Benchmark: Singlet-Excited States of Organic Molecules. J. Chem. Theory Comput. 2009, $5,2420-2435$.

(6) Laurent, A. D.; Jacquemin, D. TD-DFT Benchmarks: A Review. Int. J. Quant. Chem. 2013, 113, 2019-2039.

(7) Send, R.; Kühn, M.; Furche, F. Assessing Excited State Methods by Adiabatic Excitation Energies. J. Chem. Theory Comput. 2011, 7, 2376-2386.

(8) Kánnár, D.; Szalay, P. G. Benchmarking Coupled Cluster Methods on Valence Singlet Excited States. J. Chem. Theory Comput. 2014, 10, 3757-3765. 
(9) Jacquemin, D.; Mennucci, B.; Adamo, C. Excited-state Calculations with TD-DFT: From Benchmarks to Simulations in Complex Environments. Phys. Chem. Chem. Phys. 2011, 13, 16987-16998.

(10) Brémond, E.; Savarese, M.; Pérez-Jiménez, A. J.; Sancho-García, J. C.; Adamo, C. Speed-Up of the Excited-State Benchmarking: Double-Hybrid Density Functionals as Test Cases. J. Chem. Theory Comput. 2017, 13, 5539-5551.

(11) Pastore, M.; Mosconi, E.; De Angelis, F.; Grätzel, M. A Computational Investigation of Organic Dyes for Dye-Sensitized Solar Cells: Benchmark, Strategies, and Open Issues. J. Phys. Chem. C 2010, 114, 7205-7212.

(12) Dreuw, A.; Head-Gordon, M. Single-Reference Ab Initio Methods for the Calculation of Excited States of Large Molecules. Chem. Rev. 2005, 105, 4009-4037.

(13) Yu, H. S.; Li, S. L.; Truhlar, D. G. Perspective: Kohn-Sham Density Functional Theory Descending a Staircase. J. Chem. Phys. 2016, 145, 130901.

(14) Maitra, N. T. Perspective: Fundamental Aspects of Time-dependent Density Functional Theory. J. Chem. Phys. 2016, 144, 220901.

(15) Reisler, H.; Krylov, A. I. Interacting Rydberg and Valence States in Radicals and Molecules: Experimental and Theoretical Studies. Int. Rev. Phys. Chem. 2009, 28, 267-308.

(16) Bäppler, S. A.; Plasser, F.; Wormit, M.; Dreuw, A. Exciton Analysis of Many-body Wave functions: Bridging the Gap Between the Quasi-particle and Molecular Orbital Pictures. Phys. Rev. A 2014, 90, 052521.

(17) Luzanov, A.; Zhikol, O. In Practical Aspects of Computational Chemistry I: An Overview of the Last Two Decades and Current Trends; Leszczynski, J., Shukla, M., Eds.; Springer, 2012; pp 415-449. 
(18) In Hermitian formalisms, $\gamma^{I 0}$ is a Hermitian conjugate of $\gamma^{0 I}$, but in non-Hermitian approaches, such as coupled-cluster theory, the two are slightly different. While for quantitative interstate properties both $\gamma^{I 0}$ and $\gamma^{0 I}$ are computed, the qualitative trends in exciton properties derived from $\left(\gamma^{I 0}\right)^{\dagger}$ and $\gamma^{0 I}$ are very similar. .

(19) Plasser, F.; Wormit, M.; Dreuw, A. New Tools for the Systematic Analysis and Visualization of Electronic Excitations. Part I: Formalism. J. Chem. Phys. 2014, 141, 024106.

(20) Plasser, F.; Bäppler, S.; Wormit, M.; Dreuw, A. Visualization and Systematic Analysis of Electronic Excitations Using Density Matrices. Part II. J. Chem. Phys. 2014, 141, 024107.

(21) Tretiak, S.; Mukamel, S. Density Matrix Analysis and Simulation of Electronic Excitations in Conjugated and Aggregated Molecules. Chem. Rev. 2002, 102, 3171-3212.

(22) Luzanov, A. V.; Sukhorukov, A. A.; Umanskii, V. E. Application of Transition Density Matrix for Analysis of Excited States. Theor. Exp. Chem. 1976, 10, 354-361.

(23) Martin, R. L. Natural Transition Orbitals. J. Chem. Phys. 2003, 118, 4775-4777.

(24) Mayer, I. Using Singular Value Decomposition for a Compact Representation and Improved Interpretation of the CIS Wave Function. Chem. Phys. Lett. 2007, 437, $284-286$.

(25) Head-Gordon, M.; Grana, A. M.; Maurice, D.; White, C. A. Analysis of Electronic Transitions as the Difference of Electron Attachment and Detachment Densities. J. Phys. Chem. 1995, 99, $14261-14270$.

(26) Plasser, F.; Thomitzni, B.; Bäppler, S. A.; Wenzel, J.; Rehn, D. R.; Wormit, M.; Dreuw, A. Statistical Analysis of Electronic Excitation Processes: Location, Compactness, and Electron-Hole Correlation. J. Comp. Chem. 2015, 36, 1609-1620. 
(27) Peach, M. J. G.; Benfield, P.; Helgaker, T.; Tozer, D. J. Excitation Energies in Density Functional Theory: An Evaluation and a Diagnostic Test. J. Chem. Phys. 2008, 128, 044118 .

(28) Guido, C. A.; Cortona, P.; Mennucci, B.; Adamo, C. On the Metric of Charge Transfer Molecular Excitations: A Simple Chemical Descriptor. J. Chem. Theory Comp. 2013, 9, 3118-3126.

(29) Etienne, T.; Assfeld, X.; Monari, A. New Insight into the Topology of Excited States through Detachment / Attachment Density Matrices-Based Centroids of Charge. J. Chem. Theory Comput. 2014, 10, 3906-3914

(30) Etienne, T.; Assfeld, X.; Monari, A. Toward a Quantitative Assessment of Electronic Transitions' Charge Transfer Character. J. Chem. Theory Comput. 2014, 10, 38963905 .

(31) Le Bahers, T.; Adamo, C.; Ciofini, I. A Qualitative Index of Spatial Extent in ChargeTransfer Excitations. J. Chem. Theory Comp. 2011, 7, 2498-2506.

(32) Demoulin, B.; El-Tahawy, M. M. T.; Nenov, A.; Garavelli, M.; Le Bahers, T. Intramolecular Photoinduced Charge Transfer in Visual Retinal Chromophore Mimics: Electron Densitybased Indices at the TDDFT and PostHF Levels. Theor. Chem. Acc. 2016, 135 .

(33) Savarese, M.; Guido, C. A.; Brémond, E.; Ciofini, I.; Adamo, C. Metrics for Molecular Electronic Excitations: A Comparison between Orbital- and Density-Based Descriptors. J. Phys. Chem. A 2017, 121, 7543-7549.

(34) Plasser, F.; González, L. Communication: Unambiguous Comparison of Many-electron Wavefunctions Through Their Overlaps. J. Chem. Phys. 2016, 145, 021103. 
(35) Sneskov, K.; Christiansen, O. Excited State Coupled Cluster Methods. WIREs Comput. Mol. Sci. 2012, 2, 566.

(36) Bartlett, R. Coupled-cluster Theory and its Equation-of-motion Extensions. WIREs Comput. Mol. Sci. 2012, 2, 126-138.

(37) Krylov, A. I. Equation-of-Motion Coupled-Cluster Methods for Open-Shell and Electronically Excited Species: The Hitchhikers Guide to Fock Space. Annu. Rev. Phys. Chem. 2008, 59, 433-462.

(38) Sekino, H.; Bartlett, R. J. A Linear Response, Coupled-cluster Theory for Excitation Energy. Int. J. Quant. Chem. 1984, 26, 255-265.

(39) Geertsen, J.; Rittby, M.; Bartlett, R. J. The Equation-of-motion Coupled-cluster Method: Excitation Energies of Be and CO. Chem. Phys. Lett. 1989, 164, 57 - 62.

(40) Schirmer, J. A New Approximation Scheme for the Polarization Propagator. Phys. Rev. A 1982, 26, 2395-2416.

(41) Trofimov, A. B.; Schirmer, J. An Efficient Polarization Propagator Approach to Valence Electron Excitation Spectra. J. Phys. B 1995, 28, 2299-2324.

(42) Dreuw, A.; Wormit, M. The Algebraic Diagrammatic Construction Scheme for the Polarization Propagator for the Calculation of Excited States. WIREs Comput. Mol. Sci. 2015, 5, 82-95, doi: 10.1002/wcms.1206.

(43) Runge, E.; Gross, E. K. U. Density-Funtional Theory for Time-Dependent Systems. Phys. Rev. Lett. 1984, 52, 977-1000.

(44) Casida, M. E. In Recent Advances in Density Functional Methods Part I; Chong, D., Ed.; World Scientific: Singapore, 1995; Chapter Time-dependent Density Functional Response Theory for Molecules, pp 155-192. 
(45) Mewes, S. A.; Mewes, J.-M.; Dreuw, A.; Plasser, F. Excitons in Poly(para phenylene vinylene): A Quantum-chemical Perspective Based on High-level Ab Initio Calculations. Phys. Chem. Chem. Phys. 2016, 18, 2548-2563.

(46) Mewes, S. A.; Plasser, F.; Dreuw, A. Universal Exciton Size in Organic Polymers is Determined by Nonlocal Orbital Exchange in Time-Dependent Density Functional Theory. J. Phys. Chem. Lett. 2017, 8, 1205-1210.

(47) de Wergifosse, M.; Elles, C. G.; Krylov, A. I. Two-photon Absorption Spectroscopy of Stilbene and Phenanthrene: Excited-State Analysis and Comparison with Ethylene and Toluene. J. Chem. Phys. 2017, 146, 174102.

(48) Nanda, K.; Krylov, A. I. Visualizing the Contributions of Virtual States to Two-photon Absorption Cross-Sections by Natural Transition Orbitals of Response Transition Density Matrices. J. Phys. Chem. Lett. 2017, 8, 3256-3265.

(49) Orms, N.; Rehn, D.; Dreuw, A.; Krylov, A. I. Characterizing Bonding Patterns in Diradicals and Triradicals by Density-Based Wave Function Analysis: A Uniform Approach. J. Chem. Theory Comput. 2017, in press; ; 10.1021/acs.jctc.7b01012 .

(50) Feng, X.; Luzanov, A. V.; Krylov, A. I. Fission of Entangled Spins: An Electronic Structure Perspective. J. Phys. Chem. Lett. 2013, 4, 3845-3852.

(51) Matsika, S.; Feng, X.; Luzanov, A. V.; Krylov, A. I. What We Can Learn from the Norms of One-Particle Density Matrices, and What We Can't: Some Results for Interstate Properties in Model Singlet Fission Systems. J. Phys. Chem. A 2014, 118, $11943-11955$

(52) Shao, Y.; Gan, Z.; Epifanovsky, E.; Gilbert, A. T.; Wormit, M.; Kussmann, J.; Lange, A. W.; Behn, A.; Deng, J.; Feng, X.; Ghosh, D.; Goldey, M.; Horn, P. R.; Jacobson, L. D.; Kaliman, I.; Khaliullin, R. Z.; Kuś, T.; Landau, A.; Liu, J.; 
Proynov, E. I.; Rhee, Y. M.; Richard, R. M.; Rohrdanz, M. A.; Steele, R. P.; Sundstrom, E. J.; Woodcock III, H. L.; Zimmerman, P. M.; Zuev, D.; Albrecht, B.; Alguire, E.; Austin, B.; Beran, G. J. O.; Bernard, Y. A.; Berquist, E.; Brandhorst, K.; Bravaya, K. B.; Brown, S. T.; Casanova, D.; Chang, C.-M.; Chen, Y.; Chien, S. H.; Closser, K. D.; Crittenden, D. L.; Diedenhofen, M.; Jr., R. A. D.; Do, H.; Dutoi, A. D.; Edgar, R. G.; Fatehi, S.; Fusti-Molnar, L.; Ghysels, A.; Golubeva-Zadorozhnaya, A.; Gomes, J.; Hanson-Heine, M. W.; Harbach, P. H.; Hauser, A. W.; Hohenstein, E. G.; Holden, Z. C.; Jagau, T.-C.; Ji, H.; Kaduk, B.; Khistyaev, K.; Kim, J.; Kim, J.; King, R. A.; Klunzinger, P.; Kosenkov, D.; Kowalczyk, T.; Krauter, C. M.; Lao, K. U.; Laurent, A. D.; Lawler, K. V.; Levchenko, S. V.; Lin, C. Y.; Liu, F.; Livshits, E.; Lochan, R. C.; Luenser, A.; Manohar, P.; Manzer, S. F.; Mao, S.-P.; Mardirossian, N.; Marenich, A. V.; Maurer, S. A.; Mayhall, N. J.; Neuscamman, E.; Oana, C. M.; Olivares-Amaya, R.; ONeill, D. P.; Parkhill, J. A.; Perrine, T. M.; Peverati, R.; Prociuk, A.; Rehn, D. R.; Rosta, E.; Russ, N. J.; Sharada, S. M.; Sharma, S.; Small, D. W.; Sodt, A.; Stein, T.; Stck, D.; Su, Y.-C.; Thom, A. J.; Tsuchimochi, T.; Vanovschi, V.; Vogt, L.; Vydrov, O.; Wang, T.; Watson, M. A.; Wenzel, J.; White, A.; Williams, C. F.; Yang, J.; Yeganeh, S.; Yost, S. R.; You, Z.-Q.; Zhang, I. Y.; Zhang, X.; Zhao, Y.; Brooks, B. R.; Chan, G. K.; Chipman, D. M.; Cramer, C. J.; Goddard III, W. A..; Gordon, M. S.; Hehre, W. J.; Klamt, A.; Schaefer III, H. F.; Schmidt, M. W.; Sherrill, C. D.; Truhlar, D. G.; Warshel, A.; Xu, X.; Aspuru-Guzik, A.; Baer, R.; Bell, A. T.; Besley, N. A.; Chai, J.-D.; Dreuw, A.; Dunietz, B. D.; Furlani, T. R.; Gwaltney, S. R.; Hsu, C.-P.; Jung, Y.; Kong, J.; Lambrecht, D. S.; Liang, W.; Ochsenfeld, C.; Rassolov, V. A.; Slipchenko, L. V.; Subotnik, J. E.; Voorhis, T. V.; Herbert, J. M.; Krylov, A. I.; Gill, P. M.; Head-Gordon, M. Advances in Molecular Quantum Chemistry Contained in the Q-Chem 4 Program Package. Mol. Phys. 2015, 113, 184-215.

(53) Krylov, A. I.; Gill, P. M. W. Q-Chem: An Engine for Innovation. WIREs Comput. Mol. Sci. 2013, 3, 317-326. 
(54) Mulliken, R. Charge-Transfer Spectra. J. Chem. Phys. 1939, 7, 20.

(55) Wenzel, J.; Dreuw, A. Physical Properties, Exciton Analysis, and Visualization of Core-Excited States: An Intermediate State Representation Approach. J. Chem. Theor. Comput. 2016, 12, 1314-1330.

(56) Mewes, S.A.; Plasser, F.; Dreuw, A. Exciton Analysis in Time-Dependent Density Functional Theory: How Functionals Shape Excited-State Characters. J. Chem. Phys. 2015, 143, 171101.

(57) Stanton, J.; Bartlett, R. The Equation of Motion Coupled-cluster Method. A Systematic Biorthogonal Approach to Molecular Excitation Energies, Transition Probabilities, and Excited State Properties. J. Chem. Phys. 1993, 98, 7029-7039.

(58) Epifanovsky, E.; Zuev, D.; Feng, X.; Khistyaev, K.; Shao, Y.; Krylov, A. I. General Implementation of the Resolution-of-the-Identity and Cholesky Representations of Electron Repulsion Integrals within Coupled-Cluster and Equation-of-Motion Methods: Theory and Benchmarks. J. Chem. Phys. 2013, 139, 134105.

(59) Schäfer, A.; Huber, C.; Ahlrichs, R. Fully Optimized Contracted Gaussian Basis Sets for Atoms Lithium to Krypton. J. Chem. Phys. 1992, 97, 2571-2577.

(60) Dunning, T. H. J. Gaussian Basis Sets for Use in Correlated Molecular Calculations. I. The Atoms Boron Through Neon and Hydrogen. J. Chem. Phys. 1989, 90, 1007-1023.

(61) Kendall, R.; Dunning, T. H. J.; Harrison, R. Electron Affinities of the Firstrow Atoms Revisited. Systematic Basis Sets and Wave Functions. J. Chem. Phys. 1992, 96, 6796.

(62) Mewes, J.-M.; Herbert, J. M.; Dreuw, A. On The Accuracy of The General, StateSpecific Polarizable-Continuum Model for the Description of Correlated Ground- and Excited States in Solution. Phys. Chem. Chem. Phys. 2017, 19, 1644-1654. 
(63) Mewes, J.-M.; You, Z.-Q.; Wormit, M.; Kriesche, T.; Herbert, J. M.; Dreuw, A. Experimental Benchmark Data and Systematic Evaluation of Two a Posteriori, PolarizableContinuum Corrections for Vertical Excitation Energies in Solution. J. Phys. Chem. A 2015, 119, 5446-5464.

(64) Hirata, S.; Head-Gordon, M. Time-Dependent Density Functional Theory Within the Tamm-Dancoff Approximation. Chem. Phys. Lett. 1999, 314, 291-299.

(65) Becke, A. D. Density-Functional Thermochemistry. III. The Role of Exact Exchange. J. Chem. Phys. 1993, 98, 5648-5652.

(66) Lee, C.; Yang, W.; Parr, R. G. Development of the Colle-Salvetti Correlation-Energy Formula into a Functional of the Electron Density. Phys. Rev. B 1988, 37, 785-789.

(67) Yanai, T.; Tew, D. P.; Handy, N. C. A New Hybrid Exchange-Correlation Functional Using the Coulomb-Attenuating Method (CAM-B3LYP). Chem. Phys. Lett. 2004, 393, 51-57.

(68) Rehn, D. R. Development of Quantum Chemical Methods for Excited-State and Response Properties. Ph.D. thesis, Ruprecht-Karls University Heidelberg, 2015.

(69) Mulliken, R. Report on Notation for the Spectra of Polyatomic Molecules. J. Chem. Phys. 1955, 23, 1997.

(70) Depending on molecular orientation, symmetry labels corresponding to the same orbital or vibrational mode may be different. Q-Chem's standard molecular orientation is different from that of Mulliken. ${ }^{69}$ For example, Q-Chem places formaldehyde molecule in the $x z$-plane instead of $y z$. Consequently, for $\mathrm{C}_{2 v}$ symmetry, $b_{1}$ and $b_{2}$ labels are flipped. More details can be found at http://iopenshell.usc.edu/resources/howto/symmetry. 
(71) Foresman, J. B.; Head-Gordon, M.; Pople, J. A.; Frisch, M. J. Toward a Systematic Molecular Orbital Theory for Excited States. J. Phys. Chem. 1992, 96, 135-149.

(72) Krylov, A. I.; Sherrill, C. D.; Head-Gordon, M. Excited States Theory for Optimized Orbitals and Valence Optimized Orbitals Coupled-Cluster Doubles Models. J. Chem. Phys. 2000, 113, 6509-6527.

(73) Dreuw, A.; Weisman, J. L.; Head-Gordon, M. Long-Range Charge-Transfer Excited States in Time-Dependent Density Functional Theory Require Non-Local Exchange. J. Chem. Phys. 2003, 119, 2943-2946.

(74) Kaufmann, K.; Baumeister, W; Jungen, M. Universal Gaussian Basis Sets For an Optimum Representation of Rydberg and Continuum Wavefunctions. J. Phys. B 1989, 22, 2223-2240.

(75) Grabowski, Z. R.; Rotkiewicz, K.; Rettig, W. Structural Changes Accompanying Intramolecular Electron Transfer: Focus on Twisted Intramolecular Charge-Transfer States and Structures. Chem. Rev. 2003, 103, 3899-4032.

(76) Georgieva, I.; Aquino, A. J. A.; Plasser, F.; Trendafilova, N.; Köhn, A.; Lischka, H. Intramolecular Charge-Transfer Excited-State Processes in 4(N,NDimethylamino)benzonitrile: The Role of Twisting and the $\pi \sigma^{*}$ State. J. Phys. Chem. A 2015, 119, 62326243 .

(77) Plasser, F.; Dreuw, A. High-Level Ab Initio Computations of the Absorption Spectra of Organic Iridium Complexes. J. Phys. Chem. A 2015, 119, 1023-1036.

(78) Kautny, P.; Glöcklhofer, F.; Kader, T.; Mewes, J.-M.; Stöger, B.; Fröhlich, J.; Lumpi, D.; Plasser, F. Charge-Transfer States in Triazole Linked Donor-Acceptor Materials: Strong Effects of Chemical Modification and Solvation. Phys. Chem. Chem. Phys. 2017, 19, 18055-18067. 
(79) Bredas, J.-L.; Cornil, J.; Beljonne, D.; Dos Santos, D. A.; Shuai, Z. Excited-State Electronic Structure of Conjugated Oligomers and Polymers: A Quantum Chemical Approach to Optical Phenomena. Acc. Chem. Res. 1999, 32, 267-276.

(80) Scholes, G. D.; Rumbles, G. Excitons in Nanoscale Systems. Nat. Mater. 2006, 5, 683-696.

(81) Kirova, N. Understanding Excitons in Optically Active Polymers. Polym. Int. 2008, $57,678-688$.

(82) Tretiak, S.; Igumenshchev, K.; Chernyak, V. Exciton Sizes of Conducting Polymers Predicted by Time-Dependent Density Functional Theory. Phys. Rev. B 2005, 71, 033201.

(83) Dreuw, A.; Weisman, J. L.; Head-Gordon, M. Long-Range Charge-Transfer Excited States in Time-Dependent Density Functional Theory Require Non-Local Exchange. J. Chem. Phys. 2003, 119, 2943.

(84) Dreuw, A.; Head-Gordon, M. Failure of Time-Dependent Density Functional Theory for Long-Range Charge-Transfer Excited States: The ZincbacteriochlorinBacteriochlorin and Bacteriochlorophyll-Spheroidene Complexes. J. Am. Chem. Soc. 2004, 126, 4007-4016.

(85) Hieringer, W.; Görling, A. Failure of Time-Dependent Density Functional Methods for Excitations in Spatially Separated Systems. Chem. Phys. Lett. 2006, 419, 557-562.

(86) Wong, B. M.; Hsieh, T. H. Optoelectronic and Excitonic Properties of Oligoacenes: Substantial Improvements from Range-Separated Time-Dependent Density Functional Theory. J. Chem. Theory Comput. 2010, 6, 3704-3712.

(87) Richard, R.; Herbert, J. Time-Dependent Density-Functional Description of the ${ }^{1} L_{a}$ 
State in Polycyclic Aromatic Hydrocarbons: Charge-Transfer Character in Disguise? J. Chem. Theory Comput. 2011, 7, 1296-1306.

(88) Kuritz, N.; Stein, T.; Baer, R.; Kronik, L. Charge-Transfer-Like $\pi \rightarrow \pi^{*}$ Excitations in Time-Dependent Density Functional Theory: A Conundrum and Its Solution. J. Chem. Theory Comput. 2011, 7, 2408-2415.

(89) Colditz, R.; Grebner, D.; Helbig, M.; Rentsch, S. Theoretical Studies and Spectroscopic Investigations of Ground and Excited Electronic States of Thiophene Oligomers. Chem. Phys. 1995, 201, $309-320$.

(90) Lap, D. V.; Grebner, D.; Rentsch, S. Femtosecond Time-Resolved Spectroscopic Studies on Thiophene Oligomers. J. Phys. Chem. A 1997, 101, 107-112.

(91) Epifanovsky, E.; Kowalski, K.; Fan, P.-D.; Valiev, M.; Matsika, S.; Krylov, A. I. On the Electronically Excited States of Uracil. J. Phys. Chem. A 2008, 112, 9983-9992.

(92) Bravaya, K. B.; Krylov, A. I. On the Photodetachment from the Green Fluorescent Protein Chromophore. J. Phys. Chem. A 2013, 117, 11815-11822.

(93) Kamarchik, E.; Krylov, A. I. Non-Condon Effects in One- and Two-photon Absorption Spectra of the Green Fluorescent Protein. J. Phys. Chem. Lett. 2011, 2, 488-492.

(94) This happens because in polyatomic molecules with delocalized excited states small changes along many vibrational degrees of freedom can amount to large overall shift. For example, distributing $0.5 \mathrm{eV}$ over 50 symmetric normal modes yields $0.01 \mathrm{eV}=$ $80 \mathrm{~cm}^{-1}$ per node, which is less than a vibrational quanta for most stretching and bending vibrations.

(95) Magyar, R. J.; Tretiak, S. Dependence of Spurious Charge-Transfer Excited States on Orbital Exchange in TDDFT: Large Molecules and Clusters. J. Chem. Theory Comput. 2007, 3, 976-987. 
(96) Cave, R. J.; Zhang, F.; Maitra, N. T.; Burke, K. A Dressed TDDFT Treatment of the $2^{1} A_{g}$ States of Butadiene and Hexatriene. Chem. Phys. Lett. 2004, 389, 39-42.

(97) Knippenberg, A.; Starcke, J. H.; Wormit, M.; Dreuw, A. How Much Double Excitation Character Do the Lowest Excited States of Linear Polyenes Have? J. Chem. Phys. 2006, 329, 39-49.

(98) Hsu, C.-P.; Hirata, S.; Head-Gordon, M. Excitation Energies from Time-Dependent Density Functional Theory for Linear Polyene Oligomers: Butadiene to Decapentaene. J. Phys. Chem. A 2001, 105, 451-458.

(99) Cai, Z. L.; Sendt, K.; Reimers, J. R. Failure of Density-functional Theory and TimeDependent Density-Functional Theory for Large Extended $\pi$ systems. J. Chem. Phys. 2002, 117, 5543 .

(100) Barford, W.; Bursill, R. J.; Lavrentiev, M. Y. Density-Matrix Renormalization-Group Calculations of Excited States of Linear Polyenes. Rhys. Rev. B 2001, 63, 195108.

(101) Dallos, M.; Lischka, H. A Systematic Theoretical Investigation of the Lowest Valenceand Rydberg-excited Singlet States of Trans-butadiene. The Character of the $1^{1} \mathrm{~B}_{u}$ (V) State Revisited. Theor. Chem. Acc. 2004, 112, 16-26.

(102) Dierksen, M.; Grimme, S. Density Functional Calculations of the Vibronic Structure of Electronic Absorption Spectra. J. Chem. Phys. 2004, 120, 3544-3554.

(103) Catalán, J.; de Paz, J. L. G. On the Photophysics of All-trans Polyenes: Hexatriene Versus Octatetraene. J. Chem. Phys. 2006, 124, 034306.

(104) Angeli, C.; Pastore, M. The Lowest Singlet States of Octatetraene Revisited. J. Chem. Phys. 2011, 134, 184302. 
(105) Shao, Y.; Head-Gordon, M.; Krylov, A. I. The Spin-Flip Approach within TimeDependent Density Functional Theory: Theory and Applications to Diradicals. J. Chem. Phys. 2003, 118, 4807-4818.

(106) Bernard, Y. A.; Shao, Y.; Krylov, A. I. General Formulation of Spin-Flip Timedependent Density Functional Theory using Non-Collinear Kernels: Theory, Implementation, and Benchmarks. J. Chem. Phys. 2012, 136, 204103.

(107) Christensen, R. L.; Galinato, M. G. I.; Chu, E. F.; Howard, J. N.; Broene, R. D.; Frank, H. A. Energies of Low-Lying Excited States of Linear Polyenes. J. Phys. Chem. A 2008, 112, 12629-12636.

(108) Harbach, P. H. P.; Wormit, M.; Dreuw, A. The Third-order Algebraic Diagrammatic Construction Method (ADC(3)) for the Polarization Propagator for Closed-Shell Molecules: Efficient Implementation and Benchmarking. J. Chem. Phys. 2014, 141, 064113.

(109) Luzanov, A. V.; Casanova, D.; Feng, X.; Krylov, A. I. Quantifying Charge Resonance and Multiexciton Character in Coupled Chromophores by Charge and Spin Cumulant Analysis. J. Chem. Phys. 2015, 142, 224104.

(110) Edwards, L.; Dolphin, D. H.; Gouterman, M.; Adler, A. D. Porphyrins XVII. Vapor Absorption Spectra and Redox Reactions: Tetraphenylporphins and Porphin. J. Mol. Spectrosc. 1971, 38, 16-32.

(111) Gouterman, M. Spectra of Porphyrins. J. Mol. Spectr. 1961, 6, 138 - 163.

(112) Gouterman, M. Study of the Effects of Substitution on the Absorption Spectra of Porphin. J. Chem. Phys. 1959, 30, 1139-1161.

(113) Rubio, M.; Roos, B. O.; Serrano-Andrès, L.; Merchán, M. Theoretical Study of the Electronic Spectrum of Magnesium-Porphyrin. J. Chem. Phys. 1999, 110, 7202-7209. 
(114) Plasser, F.; Mewes, S. A.; Dreuw, A.; González, L. Detailed Wave Function Analysis for Multireference Methods: Implementation in the Molcas Program Package and Applications to Tetracene. J. Chem. Theory Comput. 2017, 13, 5343-5353. 


\section{Graphical TOC Entry}

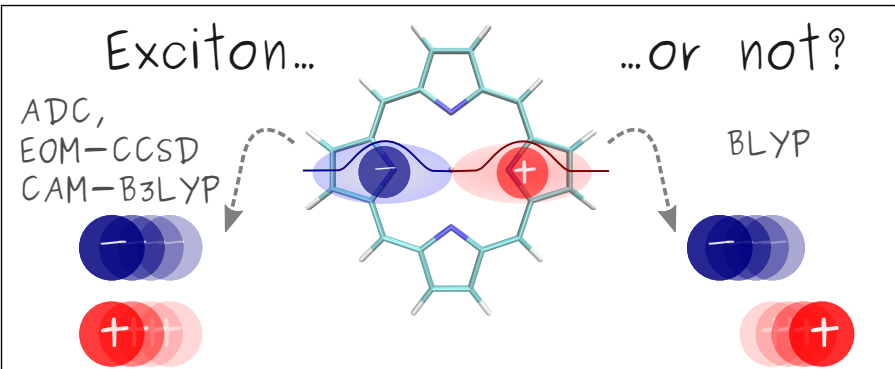

Benchmarking is an everyday task in quantum chemistry. The excitation energy is certainly an important criterion for it, but can hide discrepancy between the physical description of the underlying states by different computational protocols. Benchmarking using exciton properties provides detailed insight into excited-state description, facilitates state assignment and interpretation of results. 